\title{
Spontaneous Hydrogen Production using Gadolinium Telluride
}

Partha Kumbhakar $^{1 \ddagger}$, Arko Parui ${ }^{2 \ddagger}$, Shikha Dhakar ${ }^{3 \ddagger}$, Manas Paliwal ${ }^{1}$, Rakesh Behera ${ }^{4}$, Abhay Raj Singh Gautam ${ }^{4}$, Soumyabrata Roy ${ }^{5}$, P. M. Ajayan ${ }^{5 *}$, Sudhanshu Sharma ${ }^{3 *}$, Abhishek K. Singh $^{2^{*}}$, Chandra S. Tiwary ${ }^{1^{*}}$

${ }^{1}$ Metallurgical and Materials Engineering, Indian Institute of Technology Kharagpur, Kharagpur, India;

${ }^{2}$ Materials Research Centre, Indian Institute of Science, Bangalore, Karnataka 560012, India

${ }^{3}$ Department of Chemistry, Indian Institute of Technology Gandhinagar, Gandhinagar 382355, India;

${ }^{4}$ Materials Engineering, Indian Institute of Technology, Gandhinagar 382355, India

${ }^{5}$ Department of Materials Science and NanoEngineering, Rice University, Houston, TX 77005, USA

‡Equal contribution

*Corresponding author.: ssharma@iitgn.ac.in, abhishek@iiisc.ac.in, chandra.tiwary@metal.iitkgp.ac.in, ajayan@rice.edu

Developing a catalyst for green hydrogen production through water splitting, is one of the most promising ways to meet current energy demand. Here, we demonstrate spontaneous water splitting using gadolinium telluride (GdTe) with high hydrogen evolution rate. The spent catalyst can be reused after melting, which regains the original activity of the pristine sample. The phase formation and reusability are supported by the thermodynamics calculations. The theoretical calculation reveals ultra-low over-potential for hydrogen evolution reaction of GdTe caused by charge transfer from Te to Gd, hence enhancing the catalytic activity. Production of highly pure and instantaneous hydrogen by GdTe could accelerate fuel cell-based sustainable technologies. 
Hydrogen is a vital ingredient in chemical (majorly in synthesis of ammonia), metallurgical, glass, semiconductor, and pharmaceuticals industries ${ }^{1,2,3}$. The calculated demand for hydrogen in fossil fuels free future is expected to increase to more than two gigaton per year ${ }^{4}$. The majority of all hydrogen produced globally comes from natural gas (50\%), primarily through steam methane reforming (reaction of steam and natural gas) and the rest is generated from oil $(30 \%)$, coal $(16 \%)$, and electrolysis of water (4\%). The by-products of the major synthesis processes are complex mixtures of condensable hydrocarbons and $\mathrm{CO}_{2}$. This causes emission of $830 \mathrm{Mt}$ of $\mathrm{CO}_{2}$ per year, which is detrimental to the environment. Therefore, the instantaneous hydrogen production from water splitting will unleash the full environmental benefits.

Alkali metals like sodium, aluminum, magnesium, and potassium react with water to produce $\mathrm{H}_{2}$ gas ${ }^{5,6,7}$, However, these metals cannot be recycled, therefore they have limited scope in hydrogen production. Moreover, these reactions are highly exothermic and extremely difficult to control $^{5,6,8,9}$. Recently, several high-entropy alloy and metal-hydrides have been explored to generate $\mathrm{H}_{2}$ by controlled water splitting ${ }^{10}$. However, most of these materials have high activation barriers for $\mathrm{H}_{2}$ production. The state-of-the-art electrocatalyst consists of platinum and 2D materials hybrids that require external energy to overcome the activation barrier ${ }^{11,12,13,14,15,16}$.

Here we demonstrate instantaneous (without any external energy) generation of highly pure hydrogen using Gadolinium Telluride (GdTe) crystal from water splitting (Figure 1A-B). The GdTe is produced by a simple and easily scalable induction melting (see Method section in supplementary material). The catalyst is recyclable without loss of any activity. Post reaction analysis of catalysis confirms the formation of non-uniform oxide layers $\left(\mathrm{Gd}_{2} \mathrm{O}_{3}\right)$ which assists the reaction. Theoretical calculations indicate that the charge transfer from Te to Gd enhances the chemical activity which results in ultralow overpotential for instantaneous hydrogen generation. 
GdTe crystals produced by induction melting method, (using Gd and Te in 1:1 atomic ratio see ESI) crystallizes in the well-known NaCl-type $f c c$ structure (SG: $F m-3 m$ ) (Figure 1C) with lattice parameters of $a=b=c=6.25 \AA$ and $\alpha=\beta=\gamma=90^{\circ}$. This matches with the optimized lattice parameter of GdTe obtained from the DFT calculations and previously reported values ${ }^{17}$ (Figure S1). Synthesized sample shows four major peaks corresponding to $\mathrm{E}_{\mathrm{g}}^{1}, \mathrm{~A}_{\mathrm{g}}, \mathrm{B}_{\mathrm{g}}^{2}$, and $\mathrm{E}_{\mathrm{g}}^{2}$ modes in the Raman spectrum (Figure 1D) which confirms its purity ${ }^{18,19}$. Also, Energy dispersive X-Ray analysis (EDAX) shows the presence of Gd and Te after melting (Figure S2). Further, scanning electron microscopy (SEM) images (Inset of Figure 1C) reveal the smooth surface of the crystals.

To understand the catalytic activity of produced GdTe crystal, its ability of splitting the water to generate hydrogen without external energy is demonstrated. A large number of hydrogen bubbles were generated immediately as GdTe reacts with water at room temperature (Figure 2A and movie S1). In the beginning, a steady HER rate of $\sim 0.022 \mu \mathrm{mol} \mathrm{min} \mathrm{gm}^{-1}$ was observed for 5 min which then increases rapidly after 10 min where eventually $\sim 1.0 \mathrm{~g}$ of GdTe produced more than $\sim 1.87 \mu \mathrm{mol}$ of $\mathrm{H}_{2}$ within $30 \mathrm{~min}$ (Figure 2B). Along with water, GdTe also reacts with dry methanol and produced $\mathrm{H}_{2}$ with slower rate (Figure S3). Moreover, GdTe shows the most favorable onset potential when compared with state-of-the-art HER catalyst (Figure 2C) $20,21,22,23,24,25,26$.

Such high activity was further quantified and compared by calculating the free energies of HER for different closed packed planes of GdTe, namely (100), (110), (111) and (211), found from the experimental XRD using Computational hydrogen electrode (CHE) model by Peterson et al. ${ }^{27}$. Computed free energies of HER are compared in (Figure 2C). $\mathrm{H}^{*}$ intermediate is most strongly adsorbed on the Gd atom in the terrace site of (211) plane, with adsorption energy of $1.03 \mathrm{eV}$ where formation of $\mathrm{H}_{2}$ is highly endothermic and promotes poisoning of the surface. 
However, low stability of the intermediate on Te atom of (110) surface with adsorption energy $1.78 \mathrm{eV}$ also decreases the reactivity of GdTe. Therefore, considering all possible different adsorption sites, $\mathrm{H}^{*}$ is optimally stable on Gd atom of (100) plane having free energy of hydrogen adsorption, $\Delta \mathrm{G}_{\mathrm{H}}^{0}=0.06 \mathrm{eV}$. This outperforms Platinum $(\mathrm{Pt})$, which otherwise showed prominent capability for HER with $\Delta \mathrm{G}_{\mathrm{H}}^{0}=-0.09 \mathrm{eV}$. Similarly, the $\mathrm{Gd}$ atoms in (110) plane and corner site of (211) plane have substantial potential for HER with $\Delta \mathrm{G}_{\mathrm{H}}^{0}=0.14 \mathrm{eV}$ and $0.21 \mathrm{eV}$, respectively. Further, according to calculated overall surface activities, (100) plane shows best potential for HER reaction followed by (110), (211) and (111) surface. Eventually this ensures uninterrupted formation of large number of $\mathrm{H}_{2}$ as (100) plane has lowest surface energy of 0.02 $\mathrm{eV} / \AA^{2}$.

After 30 min of the reaction, the $\mathrm{H}_{2}$ generation reaches saturation (Figures 2A-B) and the color of the water changes from transparent to blackish brown (Inset of Figure 2A and Figure S4a). The reacted particles separated from the water after reaction saturation was found in the nanoscale range (a few nanometers) under microscopic analyses (Figure $\mathbf{S 4 b}$ ). Thus, nanostructuring of GdTe is possible by the simple addition of water without any external energy and the GdTe surface morphology changes with time and forms black-colored nanoparticles in water (Figure S4). This surface reaction is shown by in-situ optical microcopy (Figure 2E and Figure S5 and S6) and 3D surface profile of GdTe surface (Figure 2F).

Further, High-resolution TEM images (Right panel of Figure 3A) of the reacted particles separated from the water after 30 min show the formation of non-uniform amorphous oxide layer on the GdTe surface. Moreover, Fast Fourier transform (FFT) patterns of two selected regions (Inset) confirms formation of oxide layer and crystalline GdTe. EDAX also confirm the presence of Gd and Te (Figure 3B). The final product of the HER reaction after 30 minute was melted 
(GT-II sample) and its X-ray photoelectron spectroscopy (XPS), XRD was analyzed. The powder XRD pattern of GT-II sample shows that the observed peaks correspond to cubic $\mathrm{Gd}_{2} \mathrm{O}_{3}$ and $\mathrm{Gd}_{2} \mathrm{Te}_{3}$, along with GdTe (Figure 3C-D). The elemental compositions of the GT-II sample were analyzed as shown in Figure 3E-H and Figure S7. Full-scan XPS survey spectra confirm the presence of all chemical elements with their respective oxidation states (Figure S7). The high-resolution XPS spectra of Gd 4d, Gd 3d, O 1s, and Te 3d agree well with the previously reported values for pure metal and their oxidation states (Figure 3E-H). The de-convoluted spectra of Gd 4d shows three sub-peaks at $141.1 \mathrm{eV}, 143.1 \mathrm{eV}$ and $147.6 \mathrm{eV}$ corresponding to $\mathrm{Gd} 4 \mathrm{~d}_{5 / 2}$, oxidation state of $\mathrm{Gd}$ and $\mathrm{Gd}_{4} \mathrm{~d}_{3 / 2}$ respectively, indicating the presence of $\mathrm{Gd}^{3+}$ in GdTe crystal along with the formation of Gd- $\mathrm{O}_{\mathrm{x}}{ }^{28}{ }^{28}$ The de-convoluted $\mathrm{O}$ 1s spectrum shows two peaks positioned at $530.2 \mathrm{eV}$ and $531.6 \mathrm{eV}$, which could be ascribed to oxygen-metal bonds and surface adsorbed oxygen, respectively. The core level spectra of Gd 3d reveal its characteristics peaks at 1186.5 and $1218.6 \mathrm{eV}$ by virtue of $3 \mathrm{~d}_{5 / 2}$, and $3 \mathrm{~d}_{3 / 2}$, respectively which originates due to the spin-orbital coupling on a doublet. Absorption spectra also confirms the regeneration of GdTe after reaction and melting (Figure S8). Therefore, due to the presence of GdTe in GT-II sample, it regained its ability to produce $\mathrm{H}_{2}$ when reacted with water at equivalent rate. Although the amount of generated $\mathrm{H}_{2}$ decreases from freshly prepared GdTe, Further, The Te 3e XPS profile can be assigned to $\mathrm{Te}^{0}(572.9 \mathrm{eV}), \mathrm{O}-\mathrm{Te} 3 \mathrm{~d}_{3 / 2}(575.9 \mathrm{eV}), \mathrm{C}-\mathrm{Te} 3 \mathrm{~d}_{5 / 2}(583.3 \mathrm{eV})$, and O$\mathrm{Te} \mathrm{d}_{5 / 2}(586.2 \mathrm{eV}$ ) peaks (Figure 3H), indicating the formation of elemental Te and oxides of Te along with $\mathrm{Gd}_{2} \mathrm{O}_{3}$ and $\mathrm{Gd}_{2} \mathrm{Te}_{3}{ }^{29,30}$.

To understand the role of produced by-product on the reaction kinetics of GdTe in water, Thermodynamics calculations were performed at $25^{\circ} \mathrm{C}$ by varying the mole fraction of reacting reagents (Figure 3I-J). Both the production of $\mathrm{H}_{2}$ and $\mathrm{Gd}_{2} \mathrm{O}_{3}$ increase with increase in mole fraction of GdTe that reacts with 1 mole of $\mathrm{H}_{2} \mathrm{O}$. However, their production saturates at around 
0.66 mole fraction of GdTe where the amount of $\mathrm{Gd}_{2} \mathrm{Te}_{3}$ starts increasing. Further, for all increasing mole fraction of $\mathrm{H}_{2} \mathrm{O}$ that reacts with 1 mole of GdTe, production of both $\mathrm{H}_{2}$ and $\mathrm{Gd}_{2} \mathrm{O}_{3}$ increases. However, the GdTe is fully consumed at around 0.5 mole fraction $\mathrm{H}_{2} \mathrm{O}$ and the amount of Te starts increasing. This indicates that the formation of $\mathrm{Gd}_{2} \mathrm{O}_{3}$ helps in formation of $\mathrm{H}_{2}$ and drives the reaction, whereas $\mathrm{Gd}_{2} \mathrm{Te}_{3}$ and $\mathrm{Te}$ has no role. As a result, the ratio of mole fraction of GdTe and $\mathrm{H}_{2} \mathrm{O}$ should be maintained far from 1, so as to generate optimum number of products $\left(\mathrm{H}_{2}\right.$ and $\left.\mathrm{Gd}_{2} \mathrm{O}_{3}\right)$ and waste $\left(\mathrm{Gd}_{2} \mathrm{Te}_{3}\right.$ and $\left.\mathrm{Te}\right)$. Therefore, the reaction has happened in 3 possible stages: (i) After immersion, the water molecule diffuses into the crystal surface though the active site and reacts with GdTe to start a linear production of $\mathrm{H}_{2}$; (ii) after that, $\mathrm{Gd}_{2} \mathrm{O}_{3}$ is formed over the surface which opens more reaction sites progressively and reaction rate increases rapidly; (iii) At the end, the production of $\mathrm{Gd}_{2} \mathrm{O}_{3}$ and $\mathrm{H}_{2}$ reach saturation and the amount of $\mathrm{Gd}_{2} \mathrm{Te}_{3}$ is increased.

However, similar thermodynamic calculation demonstrates that while Te does not produce hydrogen, Gd does, along with the formation of stable $\mathrm{Gd}_{2} \mathrm{O}_{3}$. Therefore, to differentiate the reactivity of Gd and Te and the contribution of Te to make GdTe as an active catalyst for HER, the orbital overlap and $d$-band center model was considered. For (100), (110), and (211) surfaces, Gd atoms consist of higher amounts of states overlapping with the H states and $d$-band center closer to Fermi level (pinned at $0 \mathrm{eV}$ ) that increases the stability of $\mathrm{H}^{*}$ intermediate in comparison with Te (Figure S9-S12) and Figure 4A and Table S1. This causes Gd to atoms to react with water and form $\mathrm{Gd}_{2} \mathrm{O}_{3}$. However, 3-coordinated Te atom in (111) surface is more reactive than 6-coordinated $\mathrm{Gd}$ atom and hence, forms respective oxide. Further, the reaction of

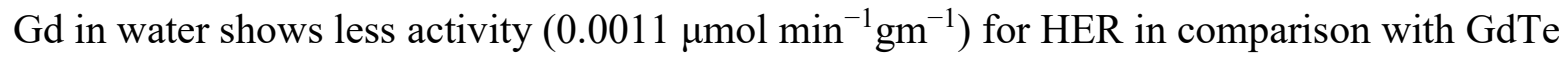
(Figure S13). As, $d$-band centre of $\mathrm{Gd}$ atom is at $-0.35 \mathrm{eV}$ for $\mathrm{Gd}(100)$ surface, which is less appropriate than the Gd atom on (100) surface of GdTe for the adsorption of hydrogen. 
Moreover, according to the DOS plot of Gd (100), the filled states near fermi level is contributed by the filled $d_{x} 2-d_{y} 2$ and $d_{x y}$ orbital. However, in GdTe (100) surface, the filled states near fermi level are mainly contributed by the $\mathrm{d}_{\mathrm{xz}}$ orbital (Figure 4B-C). This is because in GdTe the Gd atoms interact with the Te atoms by their $\mathrm{d}_{\mathrm{x}} 2-\mathrm{y} 2$ orbitals as confirmed by the electron accumulated blue region around Te atoms that is spread along the bond (Figure 4D). Correspondingly, the electron density in the $d_{x} 2-y 2$ orbital in Gd decreases as Te atoms being more electronegative pulls electron density from $\mathrm{Gd}$. Now, $\mathrm{H}$ atom approaching the surface from the z-direction interacts by its $s$-orbital with the orbitals of the surface atoms having more zdirected orientation $\left(\mathrm{d}_{\mathrm{xz}}, \mathrm{d}_{\mathrm{yz}}, \mathrm{d}_{\mathrm{z}} 2\right)$. This in turn proves the superiority of GdTe bimetallic surface over Gd surface.

In conclusion, spontaneous water splitting without any external energy was observed. One gram of GdTe crystal produced $\sim 1.75 \mu \mathrm{mol}$ of $\mathrm{H}_{2}$ within 30 minutes of vigorous reaction. The GdTe crystals were produced by industrially scalable induction melting in Argon atmosphere. The reaction produces nano structures of $\mathrm{Gd}_{2} \mathrm{O}_{3}$ on $\mathrm{GdTe}$ which assists in continuous production of hydrogen by opening more reaction sites progressively. The spent catalyst can be reused after melting which doesn't lose its catalytic activity. The theoretical calculation reveals the ultralow overpotential for HER on most stable (100) surface of GdTe as a reason of suitable orbital overlap and $d$-band center of Gd atom. Production of highly pure and instantaneous hydrogen by GdTe could accelerate fuel cell based sustainable technologies. 


\section{References}

1. Lubitz, W. \& Tumas, W. Hydrogen: An Overview. Chem. Rev. 107, 3900-3903 (2007).

2. Derycke, V. et al. Nanochemistry at the atomic scale revealed in hydrogen-induced semiconductor surface metallization. Nat. Mater. 2, 253-258 (2003).

3. Ohsawa, I. et al. Hydrogen acts as a therapeutic antioxidant by selectively reducing cytotoxic oxygen radicals. Nat. Med. 13, 688-694 (2007).

4. Oliveira, A. M., Beswick, R. R. \& Yan, Y. A green hydrogen economy for a renewable energy society. Curr. Opin. Chem. Eng. 33, 100701 (2021).

5. Mason, P. E. et al. Coulomb explosion during the early stages of the reaction of alkali metals with water. Nat. Chem. 7, 250-254 (2015).

6. Roach, P. J., Woodward, W. H., Castleman, A. W., Reber, A. C. \& Khanna, S. N. Complementary Active Sites Cause Size-Selective Reactivity of Aluminum Cluster Anions with Water. Science 323, 492-495 (2009).

7. Deal, B. E. \& Svec, H. J. Metal-Water Reactions. II. Kinetics of the Reaction between Lithium and Water Vapor. J. Am. Chem. Soc. 75, 6173-6175 (1953).

8. Shimojo, F., Ohmura, S., Kalia, R. K., Nakano, A. \& Vashishta, P. Molecular Dynamics Simulations of Rapid Hydrogen Production from Water Using Aluminum Clusters as Catalyzers. Phys. Rev. Lett. 104, 126102 (2010).

9. Jaramillo, T. F. et al. Identification of Active Edge Sites for Electrochemical H 2 Evolution from MoS 2 Nanocatalysts. Science (80-. ). 317, 100-102 (2007).

10. Sun, Y. \& Dai, S. High-entropy materials for catalysis: A new frontier. Sci. Adv. 7, (2021).

11. Zhang, J. et al. Single platinum atoms immobilized on an MXene as an efficient catalyst for the hydrogen evolution reaction. Nat. Catal. 1, 985-992 (2018).

12. Chhetri, M. et al. Mechanochemical Synthesis of Free-Standing Platinum Nanosheets and Their Electrocatalytic Properties. Adv. Mater. 27, 4430-4437 (2015).

13. Liu, D. et al. Atomically dispersed platinum supported on curved carbon supports for efficient electrocatalytic hydrogen evolution. Nat. Energy 4, 512-518 (2019).

14. Wu, Z. et al. $\mathrm{MoS}_{2}-\mathrm{MoP}$ heterostructured nanosheets on polymer-derived carbon as an electrocatalyst for hydrogen evolution reaction. J. Mater. Chem. A 6, 616-622 (2018). 
15. Cao, D. et al. Engineering the In-Plane Structure of Metallic Phase Molybdenum Disulfide via Co and O Dopants toward Efficient Alkaline Hydrogen Evolution. ACS Nano 13, 11733-11740 (2019).

16. Benck, J. D., Hellstern, T. R., Kibsgaard, J., Chakthranont, P. \& Jaramillo, T. F. Catalyzing the Hydrogen Evolution Reaction (HER) with Molybdenum Sulfide Nanomaterials. ACS Catal. 4, 3957-3971 (2014).

17. Zvoriste-Walters, C. E., Heathman, S. \& Klimczuk, T. Pressure-induced phase transitions in LnTe (Ln=La, Gd, Ho, Yb) and AmTe. J. Phys. Condens. Matter 25, 265401 (2013).

18. Treindl, A. \& Wachter, P. Raman spectra of GdS, GdSe, GdTe and LaTe: Phonon density of states in fcc metals. Phys. Lett. A 64, 147-150 (1977).

19. Lei, S. et al. High mobility in a van der Waals layered antiferromagnetic metal. Sci. Adv. 6, 1-10 (2020).

20. Mahmood, J. et al. An efficient and $\mathrm{pH}$-universal ruthenium-based catalyst for the hydrogen evolution reaction. Nat. Nanotechnol. 12, 441-446 (2017).

21. Liu, M. et al. Phosphorus-modified ruthenium-tellurium dendritic nanotubes outperform platinum for alkaline hydrogen evolution. J. Mater. Chem. A 9, 5026-5032 (2021).

22. Subbaraman, R. et al. Enhancing Hydrogen Evolution Activity in Water Splitting by Tailoring $\mathrm{Li}+$-Ni(OH)2 -Pt Interfaces. Science 334, 1256-1260 (2011).

23. Franceschini, E. A., Lacconi, G. I. \& Corti, H. R. Kinetics of the hydrogen evolution on nickel in alkaline solution: new insight from rotating disk electrode and impedance spectroscopy analysis. Electrochim. Acta 159, 210-218 (2015).

24. Oh, A. et al. Rational design of $\mathrm{Pt}-\mathrm{Ni}-\mathrm{Co}$ ternary alloy nanoframe crystals as highly efficient catalysts toward the alkaline hydrogen evolution reaction. Nanoscale 8, 1637916386 (2016).

25. Kiani, A. \& Hatami, S. Fabrication of platinum coated nanoporous gold film electrode: A nanostructured ultra low-platinum loading electrocatalyst for hydrogen evolution reaction. Int. J. Hydrogen Energy 35, 5202-5209 (2010).

26. Jiang, K. et al. Single platinum atoms embedded in nanoporous cobalt selenide as electrocatalyst for accelerating hydrogen evolution reaction. Nat. Commun. 10, 1743 (2019).

27. Peterson, A. A., Abild-Pedersen, F., Studt, F., Rossmeisl, J. \& Nørskov, J. K. How copper catalyzes the electroreduction of carbon dioxide into hydrocarbon fuels. Energy Environ. 
Sci. 3, 1311 (2010).

28. Brunckova, H. et al. XPS characterization and luminescent properties of $\mathrm{GdNbO}_{4}$ and GdTaO4 thin films. Appl. Surf. Sci. 504, 144358 (2020).

29. Li, G.-R. et al. Facile and Efficient Electrochemical Synthesis of PbTe Dendritic Structures. Chem. Mater. 20, 3306-3314 (2008).

30. Jeon, I.-Y. et al. Metalloid tellurium-doped graphene nanoplatelets as ultimately stable electrocatalysts for cobalt reduction reaction in dye-sensitized solar cells. Nano Energy 30, 867-876 (2016).

Competing interests: No conflict of interest

Supplementary Materials: Material and Methods, Computational details, Figures S1-S13 and Table S1. 


\section{FIGURES}

A

B
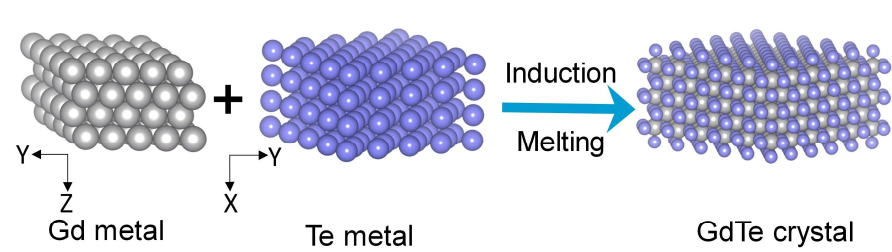

C
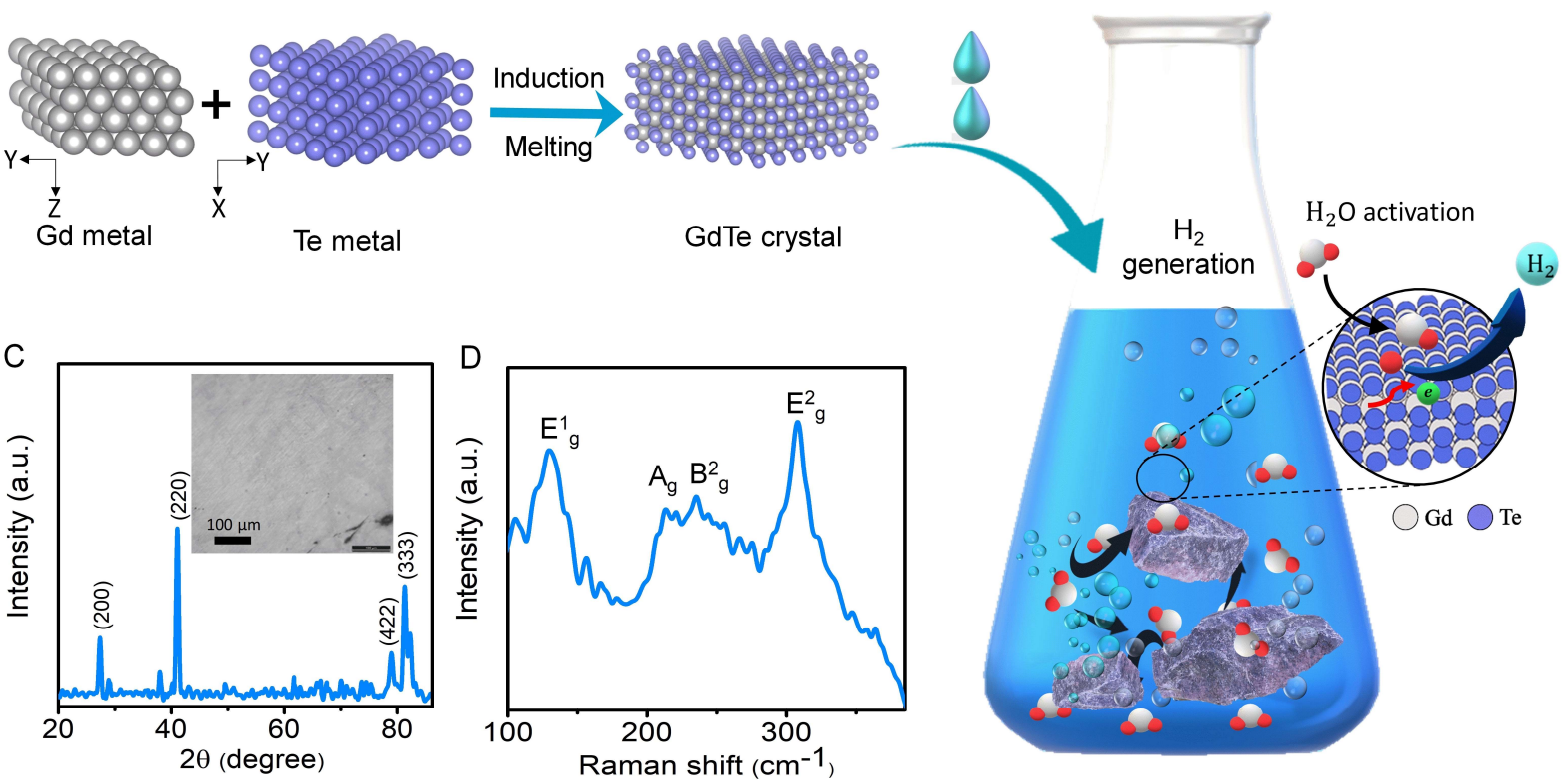

Fig. 1. Synthesis and structural property of GdTe: (A) Schematic illustration for the synthesis of GdTe crystal. Digital image with atomic arrangement of as-prepared GdTe. (B) Schematic showing representative mechanism of hydrogen evolution from GdTe surface in water. (C) XRD pattern of the as-synthesized sample. Inset shows the low magnification SEM image. (D) Raman spectrum of GdTe under excitation wavelength of $532 \mathrm{~nm}$. 


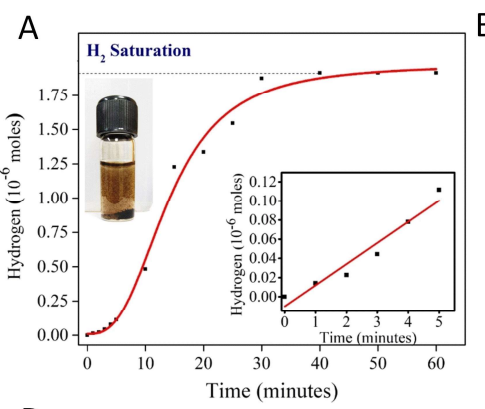

$\mathrm{D}$

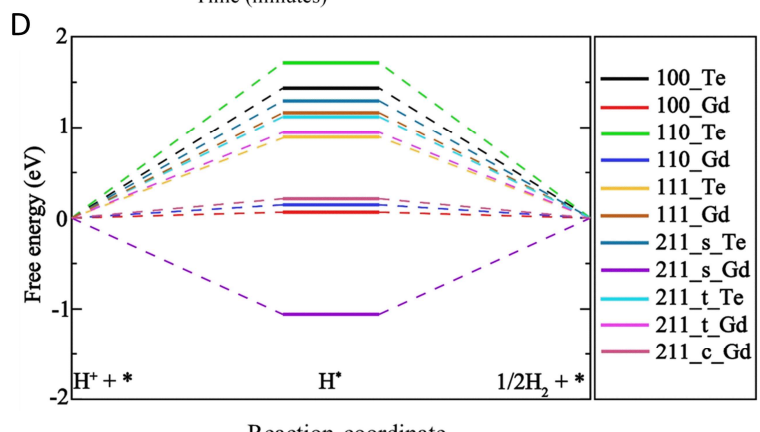

Reaction coordinate
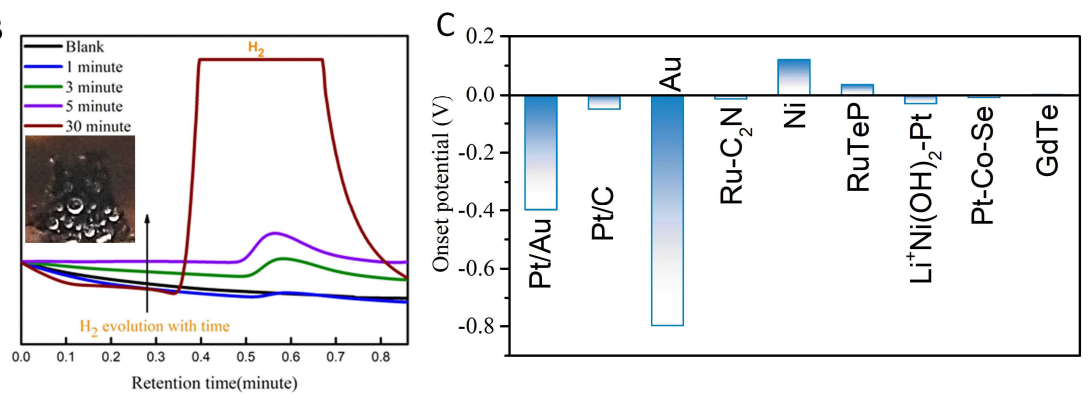

$\mathrm{E}$

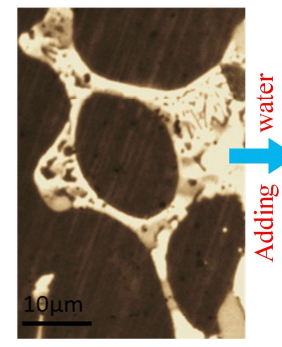

time $=0 \mathrm{~min}$

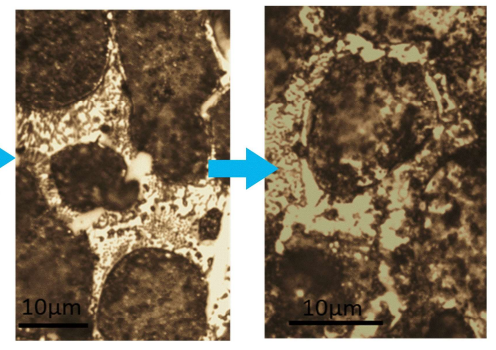

time $=2 \min$

time $=12 \mathrm{~min}$

F
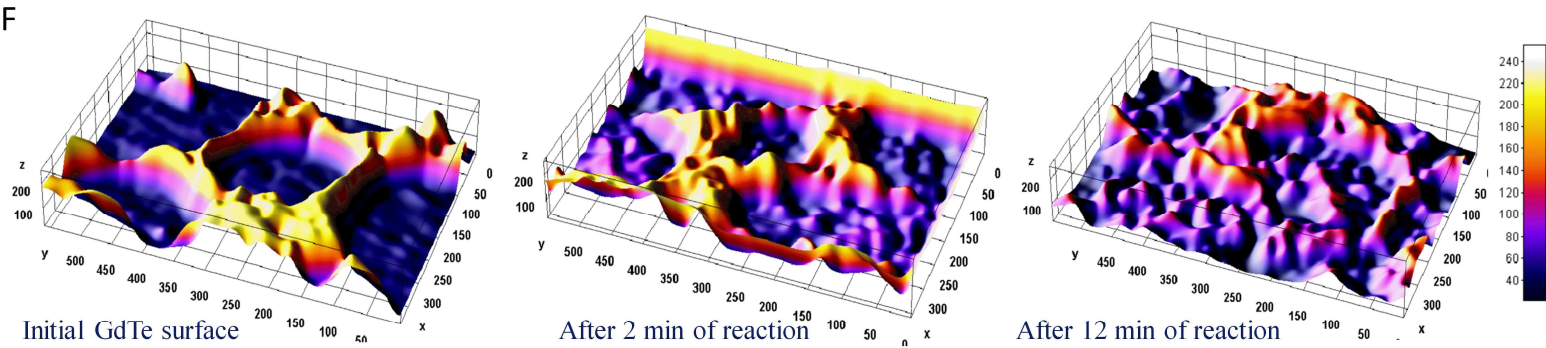

Fig. 2. Hydrogen generation and surface morphology of GdTe: (A) Hydrogen generation as a function of time. Inset shows the digital photograph of the hydrogen generation in water. (B) Represents a sample gas chromatography (GC) data. Inset shows the bubble formation during hydrogen generation (C) Comparison of onset potential with other metals and metal composites. (D) Free energy of hydrogen adsorption for different active sites. (E) Optical microscopy images of the GdTe during reaction. (F) 3D surface plots of GdTe surface during reactions. 
A

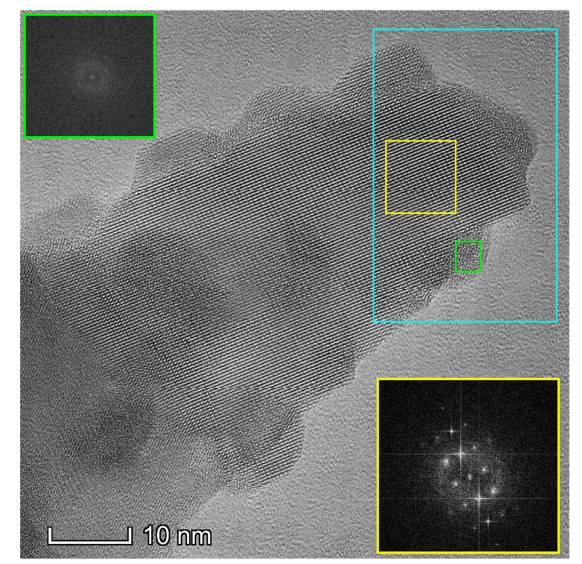

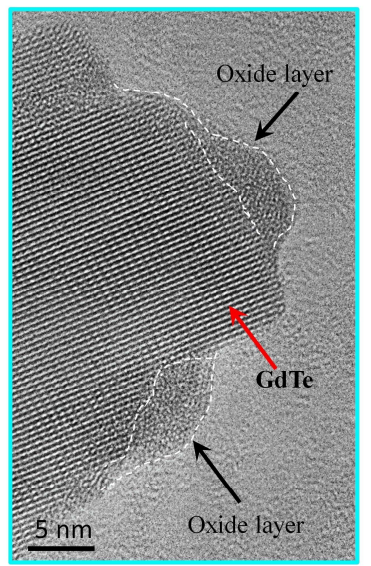
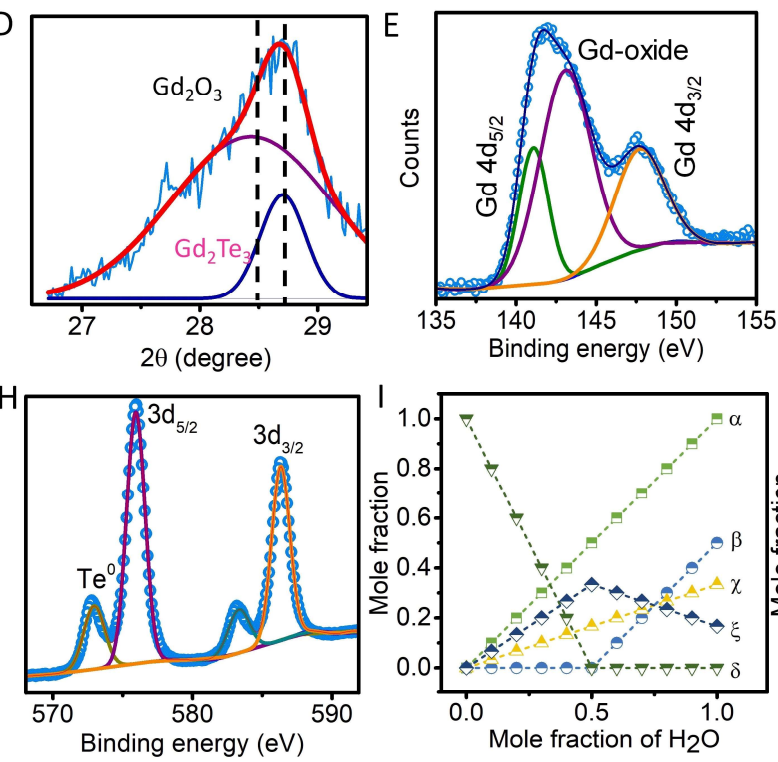

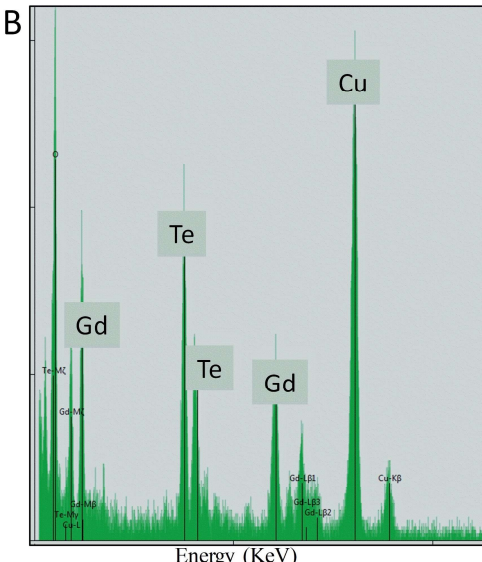

Energy $(\mathrm{KeV})$

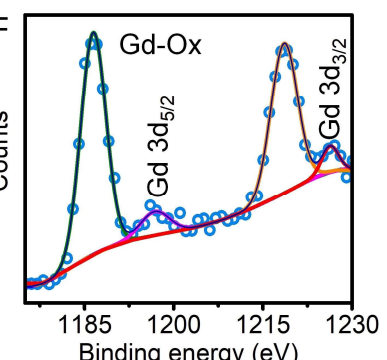

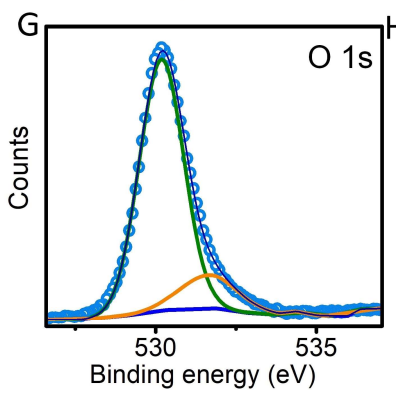

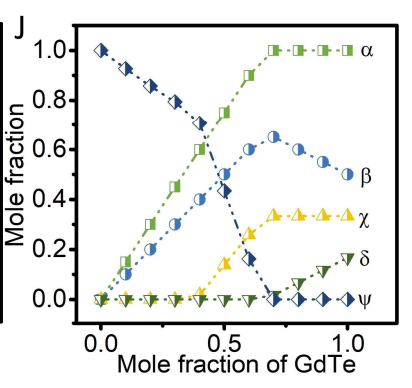

Fig. 3. Elemental analysis and thermodynamics calculations with comparison of onset potential: (A) HRTEM image of GdTe. Inset shows the FFT pattern of two different positions (Box with yellow and green color). Right panels show the enlarged view of the GdTe surface, indicates the formation of non-uniform (marked with white dash line) oxide layers on GdTe surface. (B) EDAX result of GdTe. (C) XRD pattern of chunk GdTe after reaction. (D) Multiple fitting of XRD peak and confirm the presence of $\mathrm{Gd}_{2} \mathrm{Te}_{3}, \mathrm{Gd}_{2} \mathrm{O}_{3}$. (E-H) De-convoluted XPS results of all $\mathrm{Gd}$, Te and $\mathrm{O}$ peaks. Generation of $\mathrm{H}_{2}$ gas, $\mathrm{Gd}_{2} \mathrm{Te}_{3}, \mathrm{Gd}_{2} \mathrm{O}_{3}, \mathrm{Gd}$ and $\mathrm{Te}$ (Mole fraction) of GdTe with varying molar condition of (I) GdTe and (J) water. $\alpha=\mathrm{H}_{2}$ gas (in mole), $\beta=\mathrm{Te}$ (mole), $\chi=\mathrm{Gd}_{2} \mathrm{O}_{3}$ (mole), $\delta=\mathrm{Gd}_{2} \mathrm{Te}_{3}$ (mole), $\Psi=$ Aqua components (mole), $\xi=\mathrm{GdTe}$ (mole) 


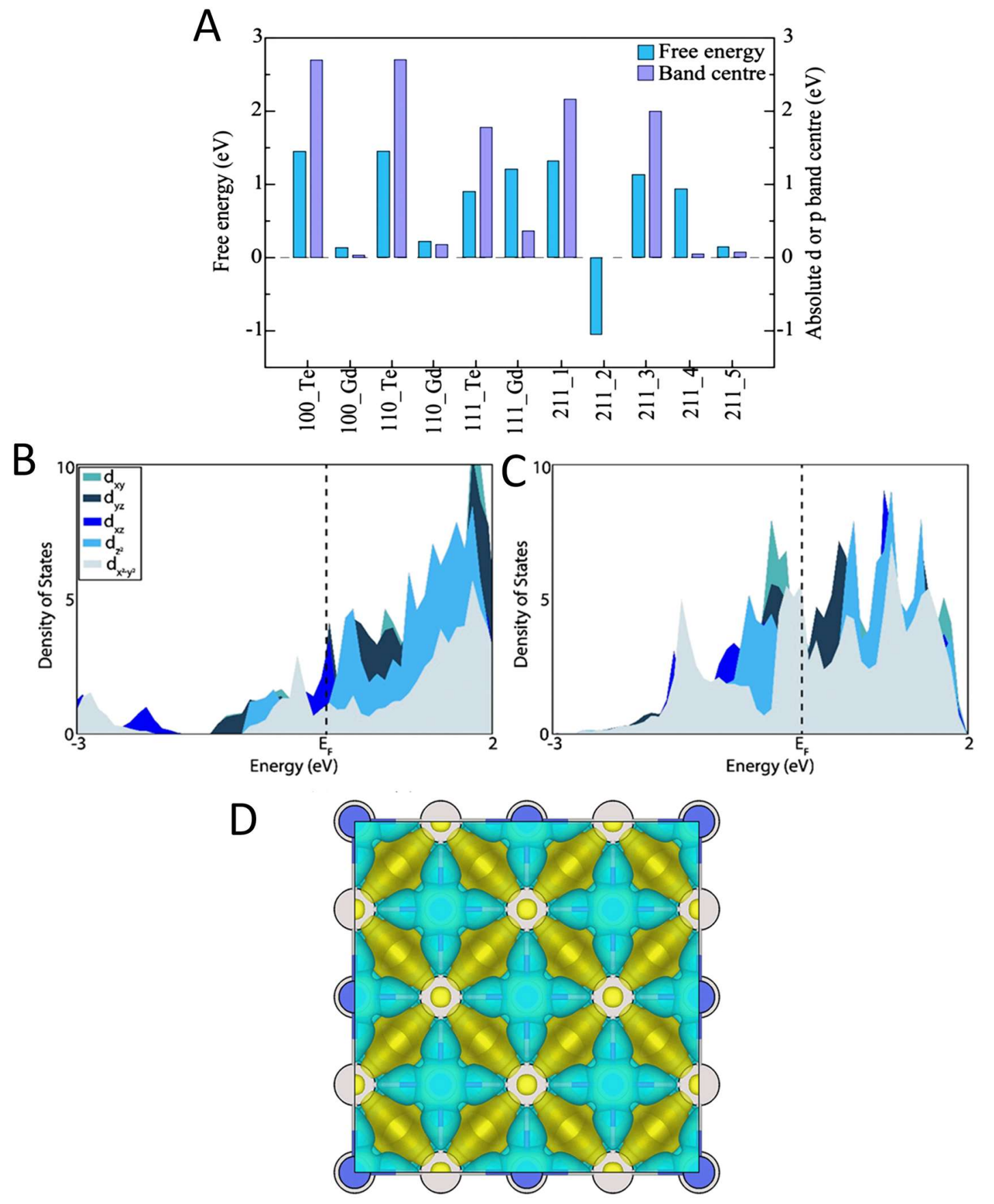

Fig. 4 DFT calculation for HER: (A) Free energy of hydrogen adsorption for different active sites. Density of states diagram of $d$-orbitals of Gd atoms on (B) (100) surface of GdTe and (C) (100) surface of Gd. (D) Charge distribution diagram on (100) surface of GdTe. Yellow region depicts charge depleted region and blue region depicts charge accumulated region. 


\title{
Supplementary Materials
}

\section{Spontaneous Hydrogen Production using Gadolinium Telluride}

Partha Kumbhakar ${ }^{1 \ddagger}$, Arko Parui ${ }^{2 \ddagger}$, Shikha Dhakar ${ }^{3 \ddagger}$, Manas Paliwal ${ }^{1}$, Rakesh Behera ${ }^{4}$, Abhay Raj Singh Gautam ${ }^{4}$, Soumyabrata Roy ${ }^{5 *}$, P. M. Ajayan ${ }^{5 *}$, Sudhanshu Sharma ${ }^{3 *}$, Abhishek K. Singh ${ }^{2 *}$, Chandra S. Tiwary ${ }^{1}$

${ }^{1}$ Metallurgical and Materials Engineering, Indian Institute of Technology Kharagpur, Kharagpur, India;

${ }^{2}$ Materials Research Centre, Indian Institute of Science, Bangalore, Karnataka 560012, India

${ }^{3}$ Department of Chemistry, Indian Institute of Technology Gandhinagar, Gandhinagar 382355, India;

${ }^{4}$ Materials Engineering, Indian Institute of Technology, Gandhinagar 382355, India

${ }^{5}$ Department of Materials Science and NanoEngineering, Rice University, Houston, TX 77005, USA

\# Equal contribution

This file includes:

\author{
Materials and Methods \\ Supplementary Text \\ Figs. S1 to S13 \\ Tables S1 \\ Supplementary Movie S1
}




\section{Materials and Methods}

\section{Synthesis of GdTe:}

Gadolinium telluride (GdTe) was obtained via the vacuum induction melting route. Stoichiometric amounts of $99.99 \%$ pure gadolinium and tellurium were taken in a quartz tube. The metals were melted at a temperature of $1500{ }^{\circ} \mathrm{C}$ under vacuum conditions and cooled by quenching to room temperature using Argon. Although the melting point of $\mathrm{GdTe}$ is $1825{ }^{\circ} \mathrm{C}$, a lower temperature was sufficient because the high vacuum conditions of $1 \times 10^{-5} \mathrm{mbar}$ inside the furnace reduces the melting point of the metals and the compound. Hydrogen evolution reaction started immediately after contact with the atmosphere. Thus, the samples were stored under a vacuum. While handling the samples, they were immersed in ethanol to reduce interaction with ambient water vapor.

\section{Characterizations:}

The GdTe polycrystals obtained were powdered and XRD patterns were obtained using a Bruker D8 Advance Diffractometer with $\mathrm{Cu}-\mathrm{K} \alpha(\lambda=1.5406 \AA)$ radiation and characterized based on the Pearson's Crystal Database. Room temperature Raman spectroscopy was done using WITec UHTS Raman Spectrometer (UHTS 300 VIS, Germany) at a laser excitation wavelength of 532 nm. UV-Visible Spectroscopy was used to study the optical absorbance.

\section{HER measurements:}

The catalyst was enclosed in a glass vial, and gas inside the vial was taken as a blank gas sample. Deionized water $(1 \mathrm{ml})$ was added to the glass vial, and gas evolution was analyzed in a specified time interval. GdTe reacted with water immediately at room temperature and produced gas bubbles on the surface of the GdTe sample in the glass vial. Quantitative analyses of the product were carried out to corroborate the hypothesis of Hydrogen production and to determine 
the number of moles of gas produced. High precision gas-tight syringe was used to transfer the evolved gases into a gas chromatograph (GC; Dhruva CIC Baroda) during the experiment. Thermal Conductivity Detector (TCD) was used for $\mathrm{H}_{2}$ detection and quantification. The Standard gas mixture was used for GC Calibration. $1 \mathrm{gm}$ of GdTe produced more than $1.87 \mu \mathrm{mol}$ of Hydrogen within 30 minutes. The $\mathrm{H}_{2}$ generation reaches saturation within $30 \mathrm{~min}$, whereas for $\mathrm{Gd}$ in water, Hydrogen production occur but comparatively very less to GdTe and didn't attend saturation even after 1380 minutes (23 hours) of experiment.

We also note at the early stage, around $\sim 5$ min, during which the $\mathrm{H}_{2}$ evolution rate is linearly increased. We observed

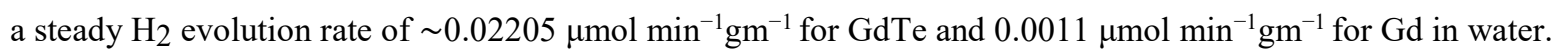

\section{Computational Details:}

Density functional theory (DFT) were done with Vienna ab initio simulations (VASP) package (1). The electron-ion interactions were described using all-electron projector augmented wave pseudopotentials, and Perdew-Bruke-Ernzehof (PBE) generalized gradient approximation (GGA) was used to approximate the electronic exchange and correlations (2). The plane-wave kinetic energy cut off of $520 \mathrm{eV}$ was used. All the structures were optimized using a conjugate gradient scheme until the energies, and the components of forces reached $10^{-6} \mathrm{eV}$ and $0.001 \mathrm{eV} \AA^{-}$ ${ }^{1}$ for bulk GdTe and $10^{-5}$ and $0.01 \mathrm{eV} \AA^{-1}$ for all the surfaces respectively. A vacuum region of 10 $\AA$ was added in the z-direction to prevent interactions between the periodic images. The Brillouin zone of bulk GdTe, (100), (110), (111), and (211) planes were sampled with $7 \times 7 \times 7$ MonkhorstPack, $3 \times 3 \times 1$ Monkhorst-Pack, $3 \times 3 \times 1$ Monkhorst-Pack, $3 \times 3 \times 1$ Monkhorst-Pack, $2 \times 3 \times 1$ Monkhorst-Pack, respectively. Density of states (DOS) calculations for planes were performed by sampling the Brillouin zone with $7 \times 7 \times 1$ Monkhorst-Pack. Band center was calculated by 
considering the first moment of d-states for $\mathrm{Gd}$ atoms and $\mathrm{p}$ - states for Te atoms. All the calculations were spin-polarized.

Posterior to the electronic and lattice optimization of the bulk GdTe structure, taken from Materials Project database (3), different surfaces, namely (100), (110), (111) and (211) were created each of them having 4 layers. Afterwards, $2 \times 2 \times 1$ supercell of each considered surfaces was created followed by electronic optimization. (100), (110), and (111) surfaces can adsorb H on either Gd or Te atom. However, for (211) surface, it can allow H to adsorb on 5 different positions, namely Gd and Te atoms on step, terrace and only Gd atom on corner site as adsorbed $\mathrm{H}$ on $\mathrm{Te}$ atom diffuses on top of Gd atom after optimization.

HER consist of two elementary steps:

$$
\begin{gathered}
*+\mathrm{H}^{+}+\mathrm{e}^{-} \rightarrow \mathrm{H}^{*} \\
\mathrm{H}^{*}+\mathrm{H}^{+}+\mathrm{e}^{-} \rightarrow *+\mathrm{H}_{2}
\end{gathered}
$$

The adsorption energy of the $\mathrm{H}^{*}$ intermediate involved in HER was calculated from the DFT using the following expression $(27,28)$ :

$$
\Delta \mathrm{E}_{\mathrm{ads}}=\mathrm{E}_{\mathrm{H}^{*}}-\mathrm{E}_{*}-\frac{1}{2} \mathrm{E}_{\mathrm{H}_{2}}
$$

where, * represents a pristine GdTe surface, and the * superscript denotes that hydrogen is adsorbed on the GdTe surface. The intermediate adsorption energy was converted into Gibb's free energy of adsorption using the following $(4,5)$ :

$$
\Delta \mathrm{G}_{\mathrm{ads}}=\Delta \mathrm{E}_{\mathrm{ads}}+\Delta \mathrm{ZPE}-\mathrm{T} \Delta \mathrm{S}-\Delta \mathrm{G}_{\mathrm{U}}
$$

where, $\triangle \mathrm{ZPE}$ is the difference in zero-point energies, $\Delta \mathrm{S}$ is the change in entropy due to vibrational contributions, and $\Delta \mathrm{G}_{\mathrm{U}}=-\mathrm{e}_{\mathrm{U}}$, where $\mathrm{U}$ is the applied electrode potential. The values of ( $\Delta \mathrm{ZPE}-$ $\mathrm{T} \Delta \mathrm{S})$ for the reference molecule $\left(\mathrm{H}_{2}\right.$ gas $)$ is $0.1328 \mathrm{eV}$. Calculated values of adsorption energies, $(\Delta \mathrm{ZPE}-\mathrm{T} \Delta \mathrm{S})$, free energies of hydrogen adsorption are given in Table $\mathbf{S 1}$. 
The goal is to find optimal stability of $\mathrm{H}^{*}$ on the surface that agrees with the Sabatier principle. Hinnemann et al. confirmed that $\Delta \mathrm{G}_{\mathrm{H}}^{0} \approx 0$ is a good descriptor for materials acting as catalyst for hydrogen evolution (6).

The overall surface activitiy is given by $\frac{\sum_{n} \Delta G_{H}^{0}(n)}{\sum_{n} n}$, where $\sum_{n} \Delta G_{H}^{0}(n)$ is the total free energy of hydrogen adsorption in (n) number of different sites for a particular surface

Band centre and DOS have already been established to be good descriptors for correlating materials and their HER catalytic activity. As the number of states of a particular adsorption site increases in the energy range of $\mathrm{H}$ states, interaction between them rises and consequently, adsorption energy increases.

The d-band centre model has been used extensively to understand the role of d-electrons in bonding with the adsorbates by Norskov et. al. (7). According to this model, the d-band centre of the adsorbent being in close in energy with respect to the Fermi energy enhances the bond stability with adsorbate, having more electrons in the bonding state and less electrons in the bonding state the antibonding state, as shown in Table S1.

In order to ascertain the feasibility of $\mathrm{H}_{2}$ production owing to chemical reaction of GdTe with water, thermodynamic calculations were performed using FactSage 8.0 software using FactPS database. The thermodynamic properties of the reactants (GdTe and water) and all the possible reaction products are already present in the database. The calculations predict the reaction products on the basis of the global minimization of system's free energy depending on pressure and temperature. 
(a)

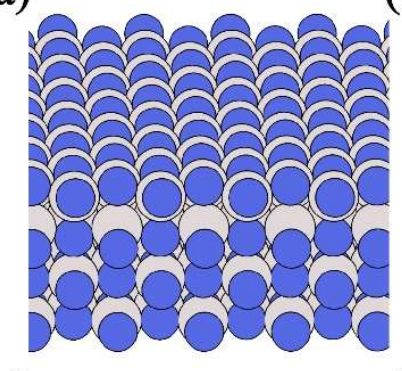

(e)

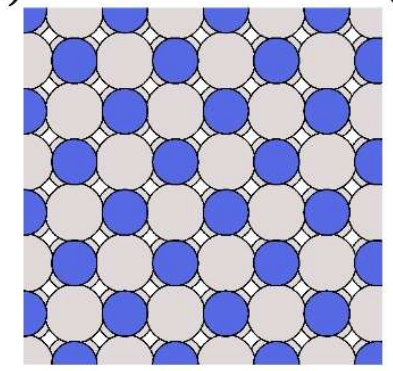

(b)

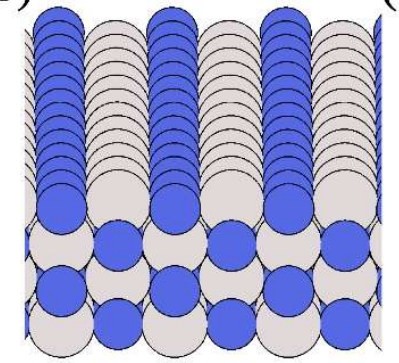

(f)

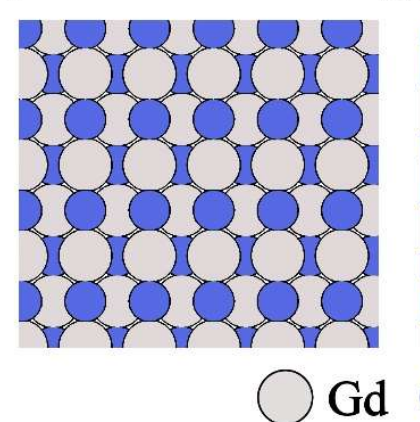

(c)

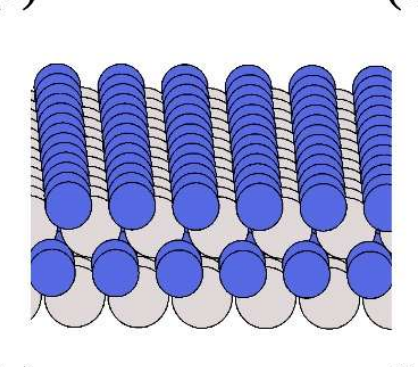

(g)

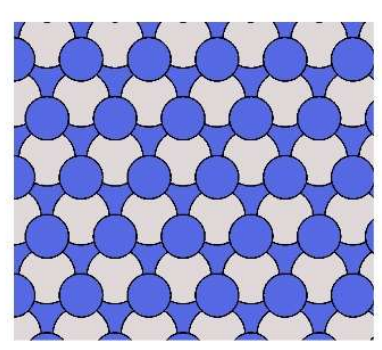
$\mathrm{Te}$ (d)

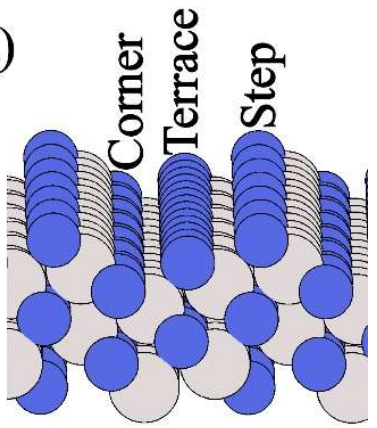

(h)

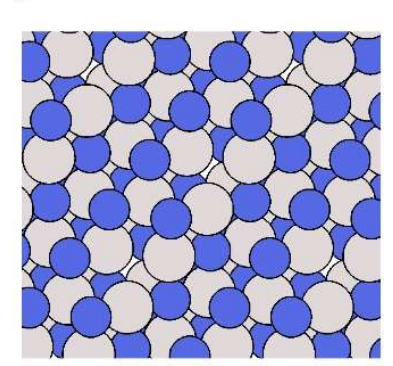

Figure S1. (a), (b), (c), (d) represents the side view and (e), (f), (g), (h) represents the top view of (100), (110), (111) and (211) surfaces, respectively 


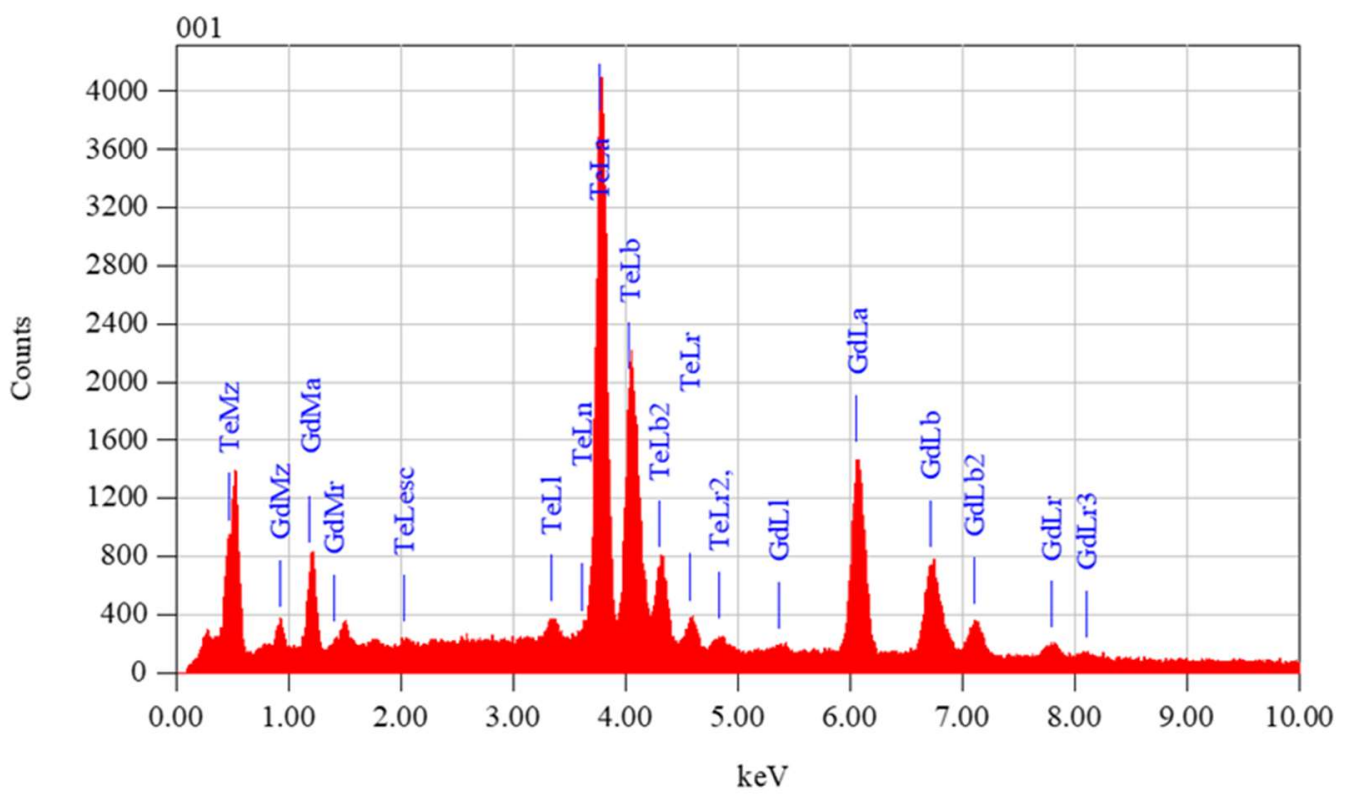

Figure S2. EDAX spectra of synthesized GdTe sample 

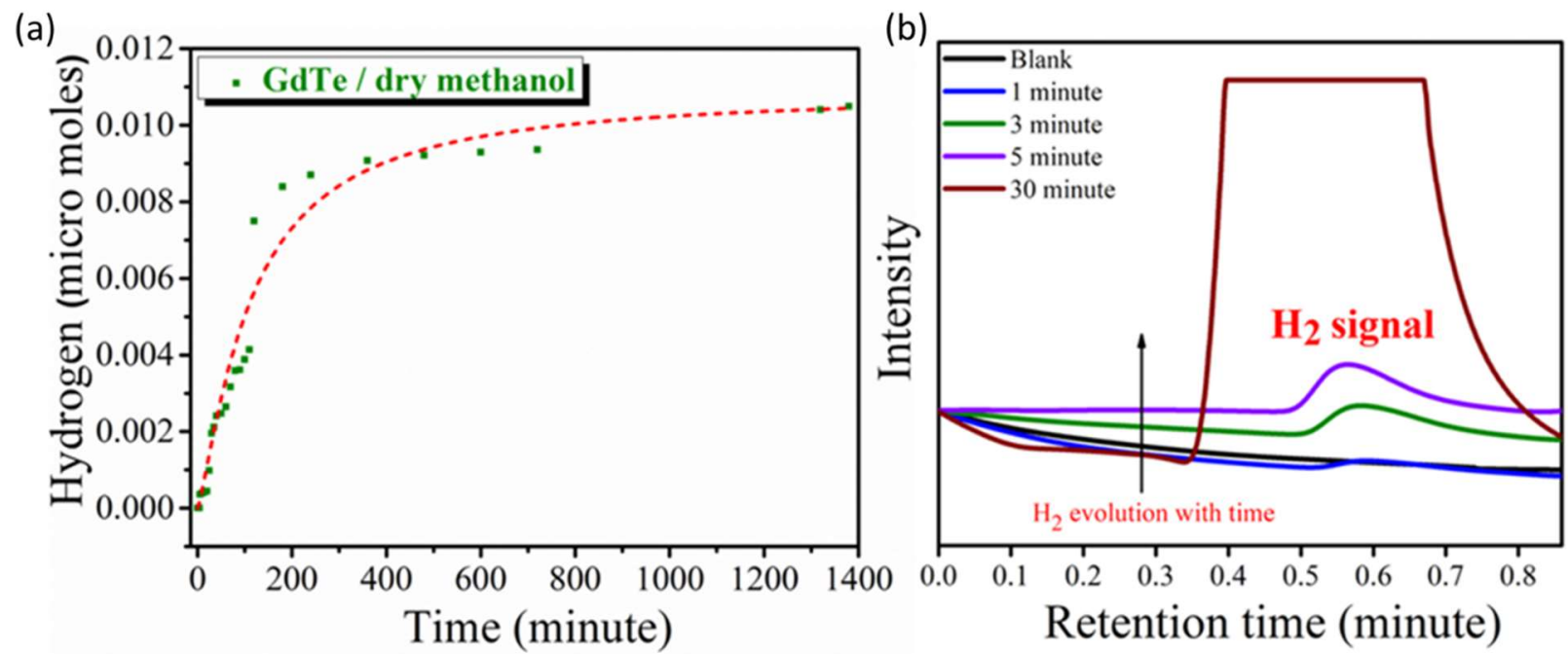

Figure S3: (a) Hydrogen generation as a function of time for GdTe in dry methanol. A Logistic fit of the data recorded is shown as red dashed line. (b) Sample Gas Chromatography data representing the generation of $\mathrm{H}_{2}$ over period of time for GdTe in water. 
A

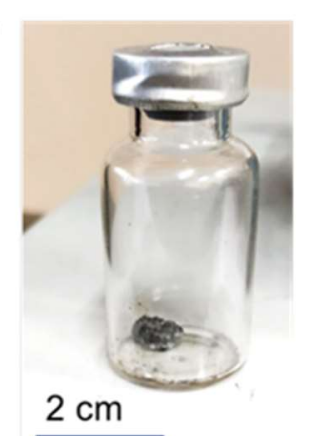

Before

Colour change during reaction

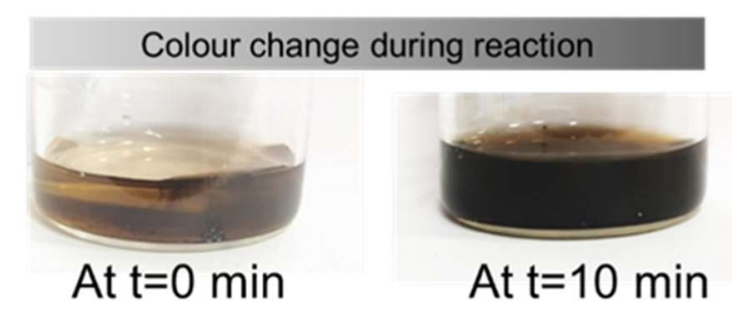

B

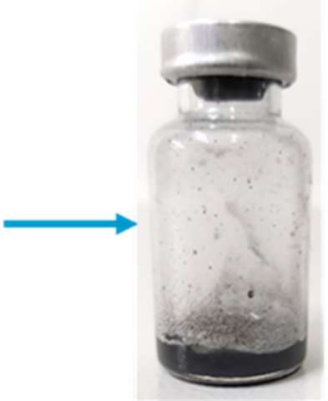

After

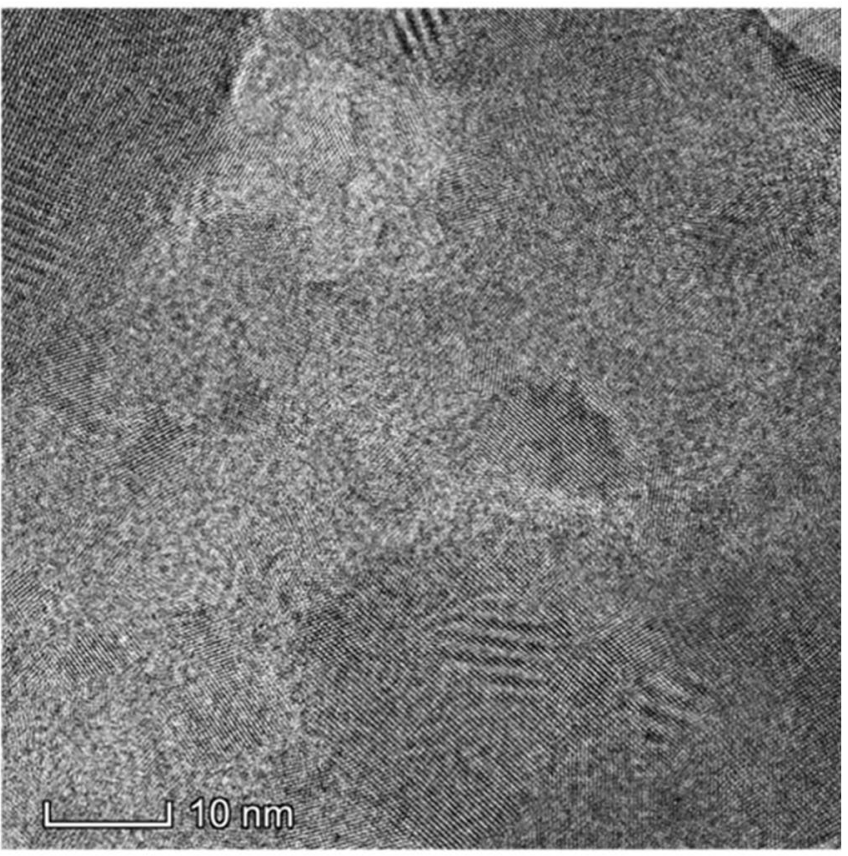

Figure S4. (a) Digital photographs of GdTe before and after reaction. (b) HRTEM image of the GdTe. 

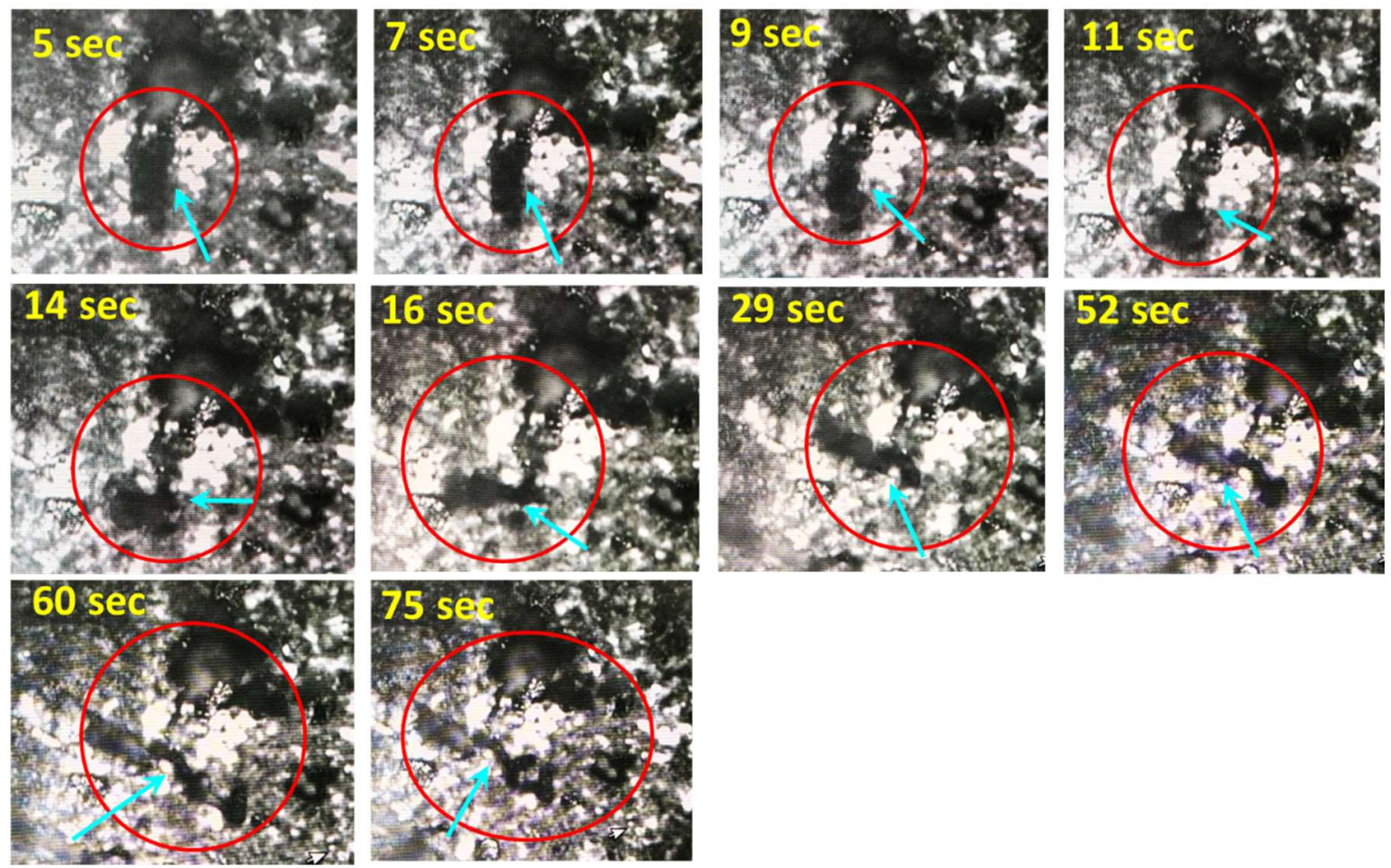

Figure S5. Snapshots of change of GdTe surface during reaction with water. Red circles indicate the changes. 


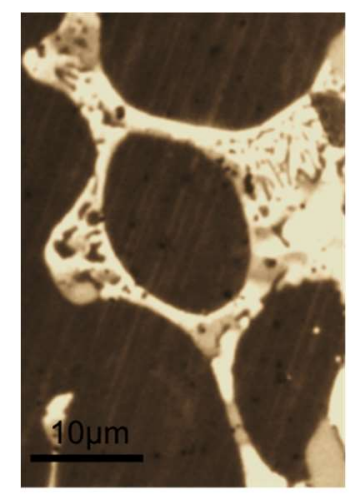

Initial $\mathrm{T}=\mathbf{0} \min$
Change of GdTe surface with reaction time
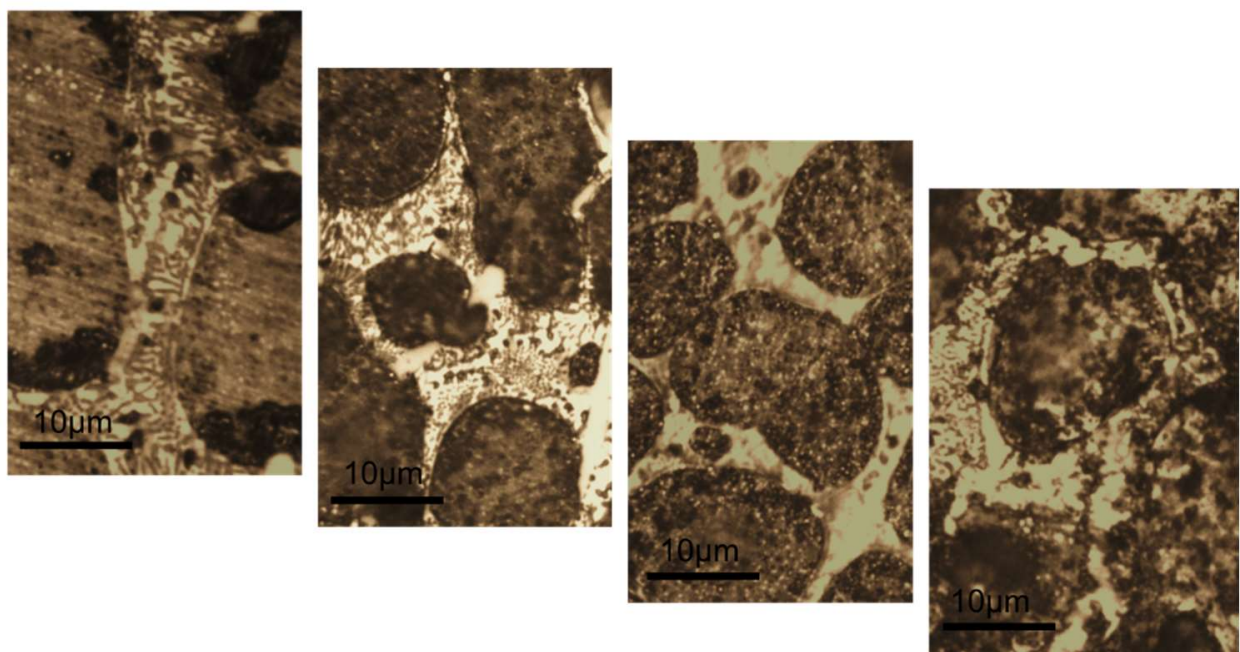

Figure S6. Optical microscopy images of the GdTe surface as a function of time. 

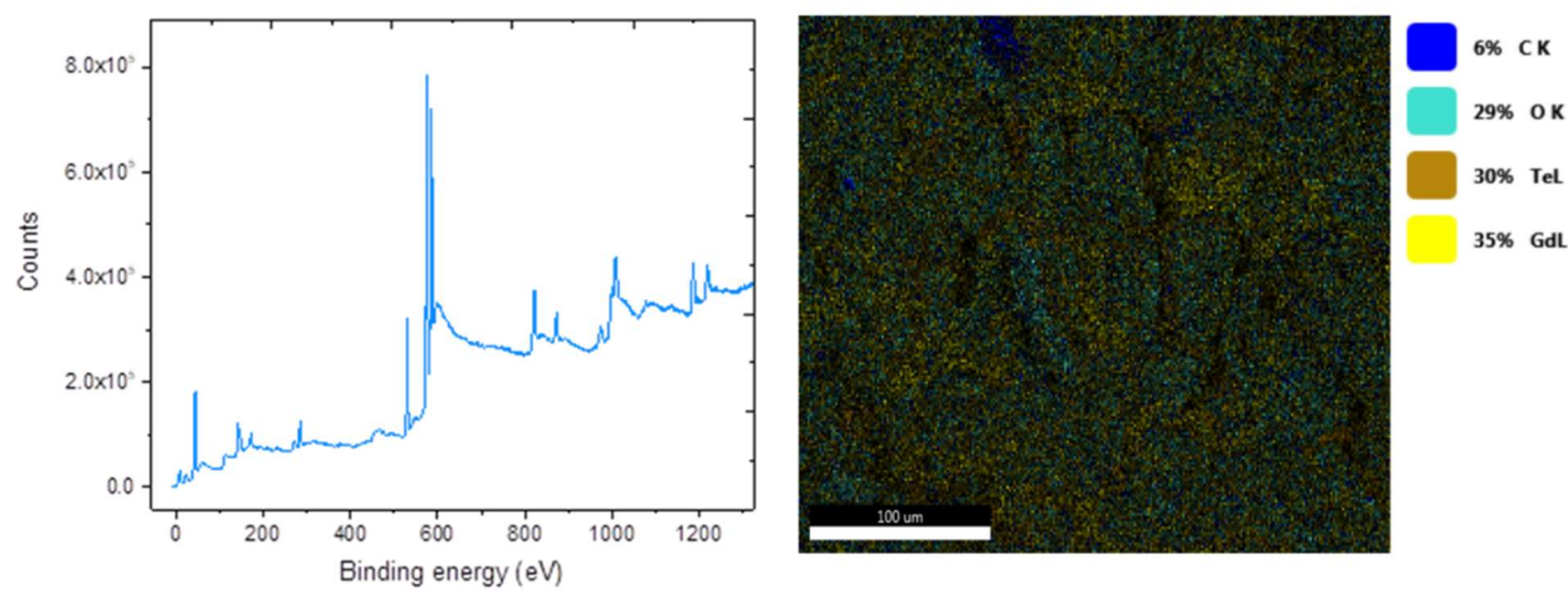

Figure S7. Full scan XPS spectrum of GdTe and elemental mapping of the surface. 

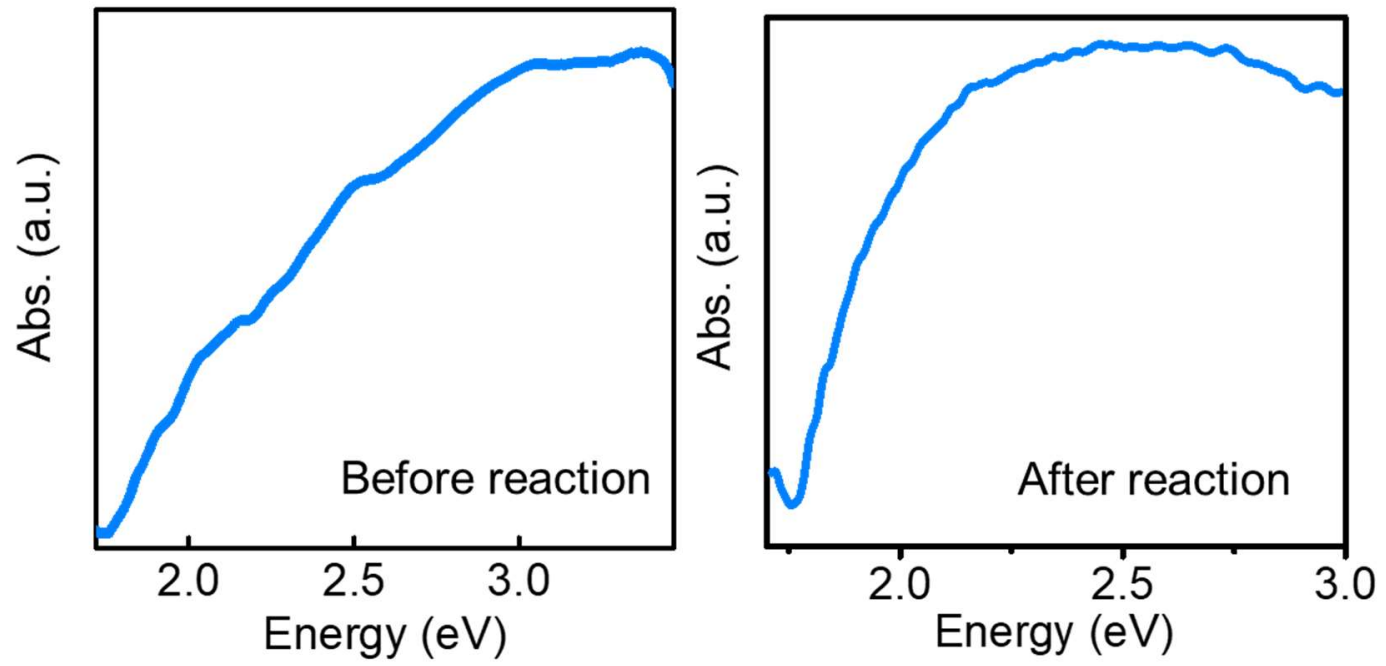

Figure S8: Optical absorption spectra of GdTe before and after HER reaction 
(a)

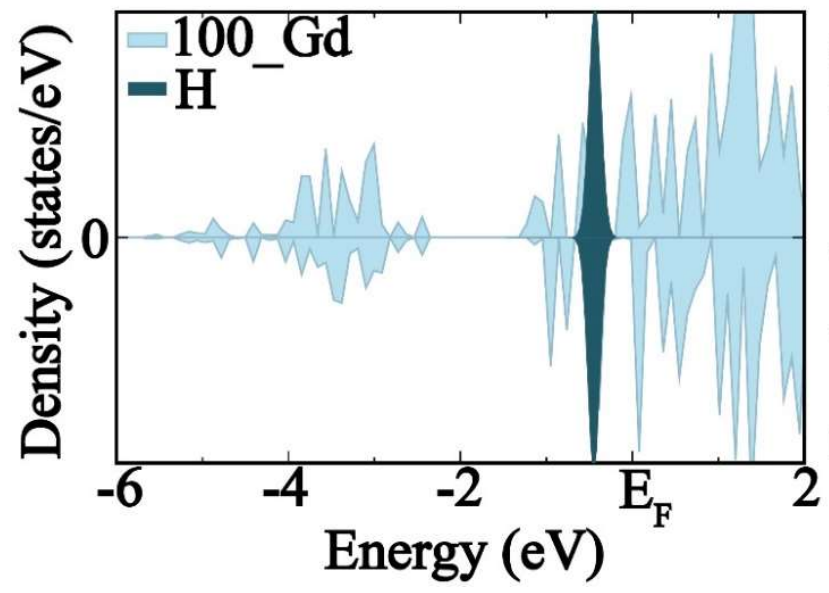

(b)

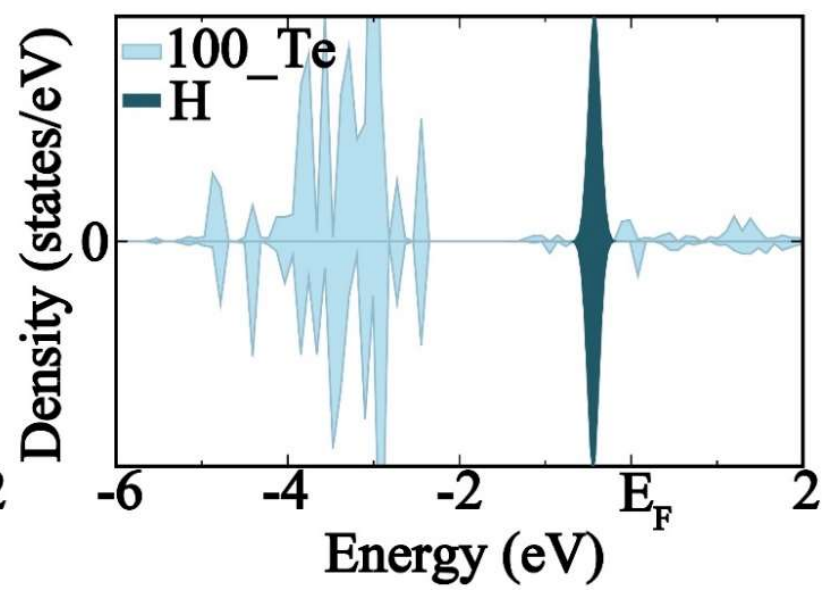

Figure S9. Partial density of states(DOS) for Gd and Te atom on (100) surface

(a)

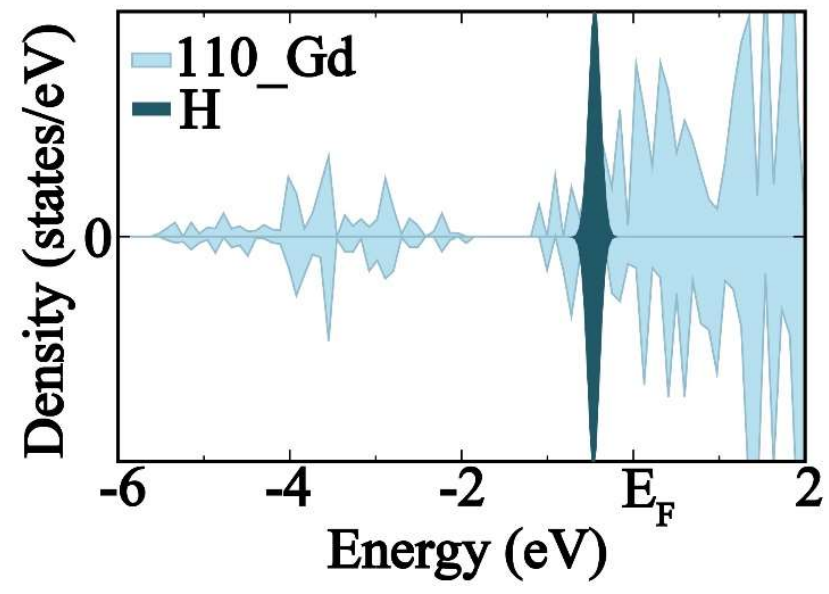

(b)

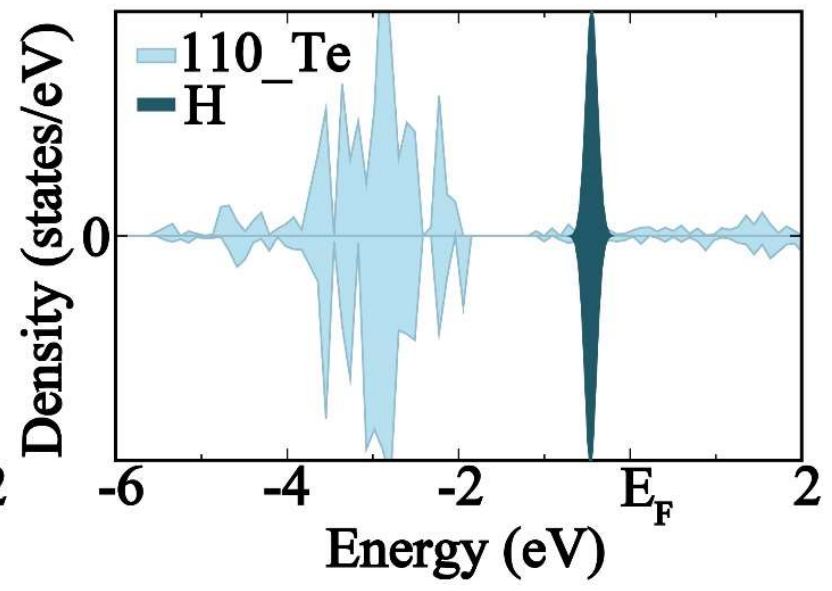

Figure S10: Partial density of states(DOS) for Gd and Te atom on (110) surface 
(a)

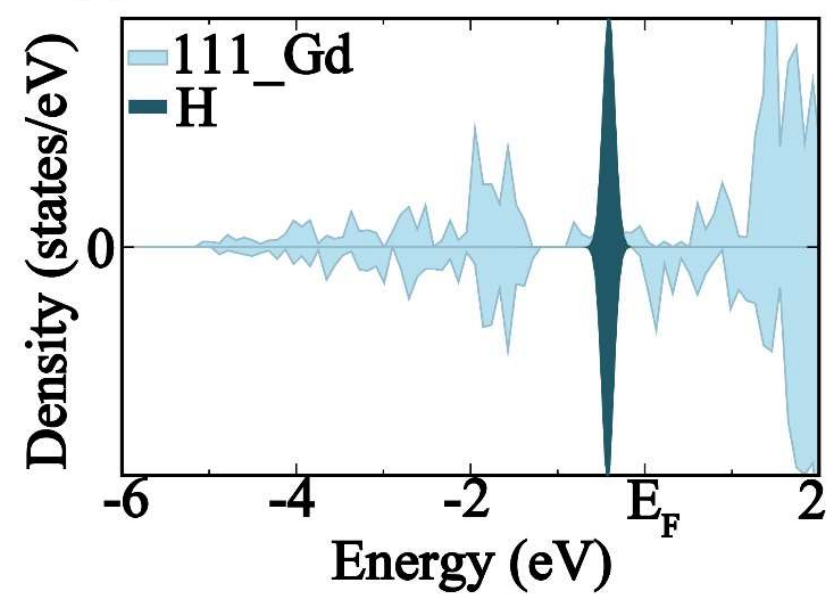

(b)

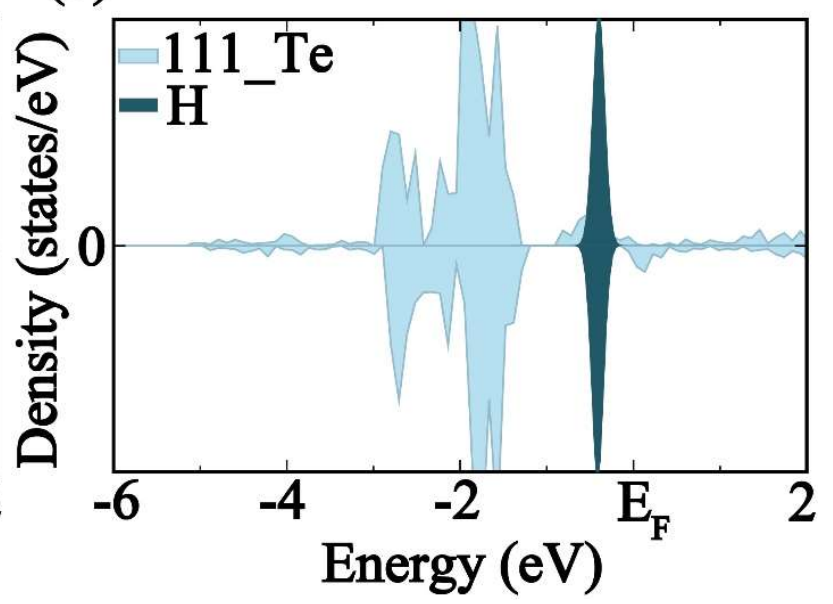

Figure S11: Partial density of states(DOS) for Gd and Te atom on (111) surface 
(a)

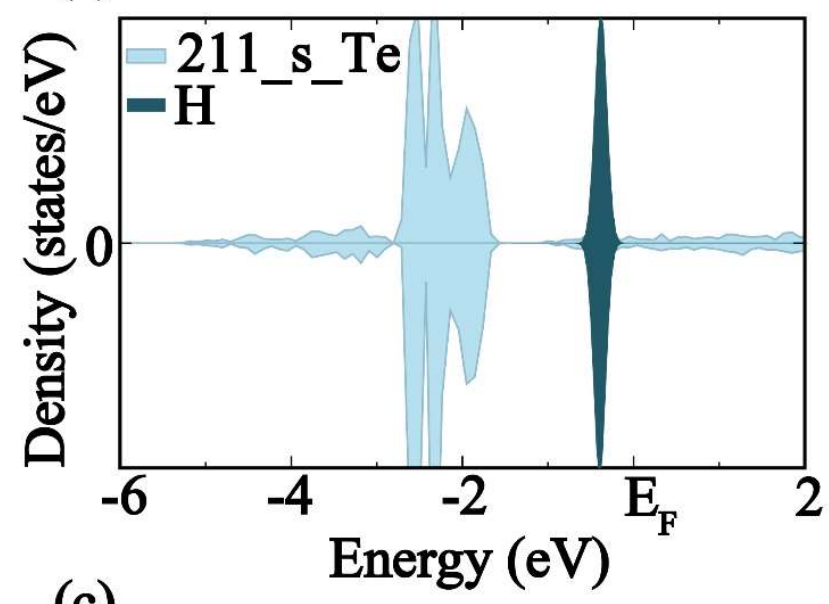

(c)

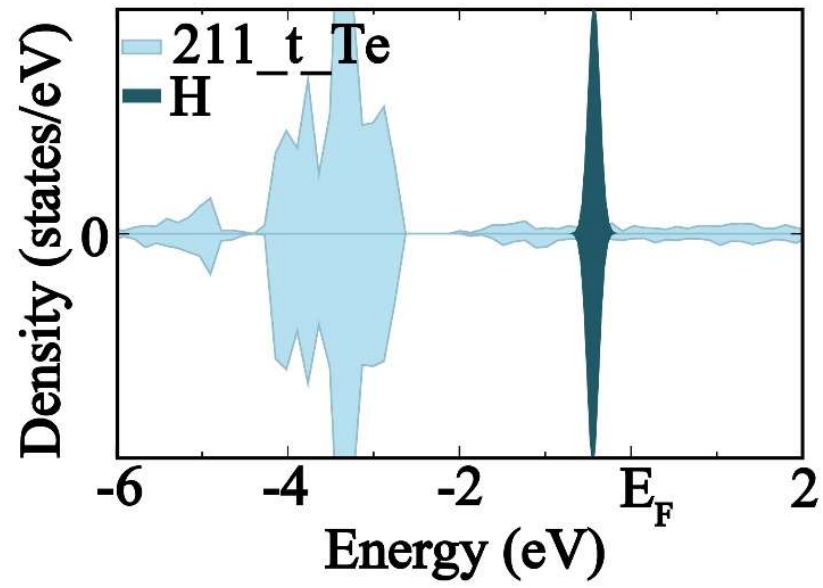

(b)

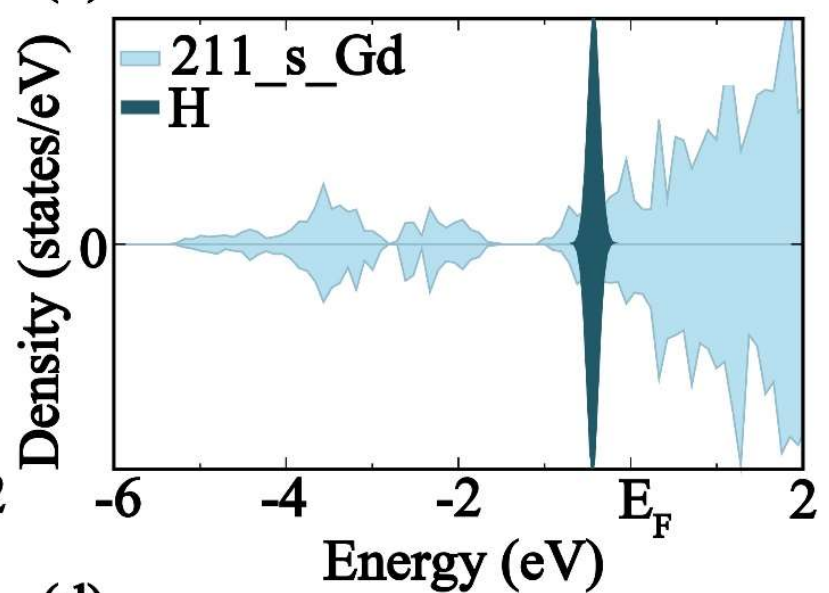

(d)

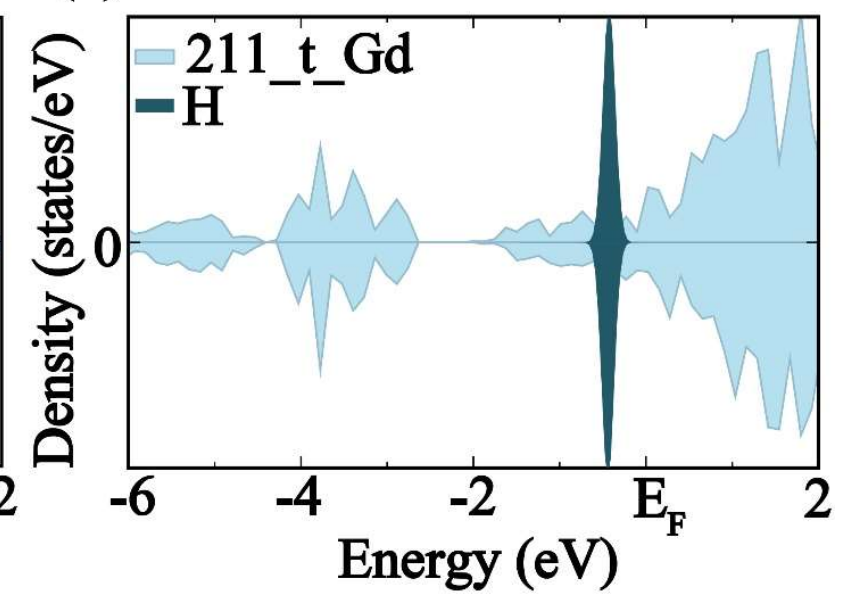

(e)

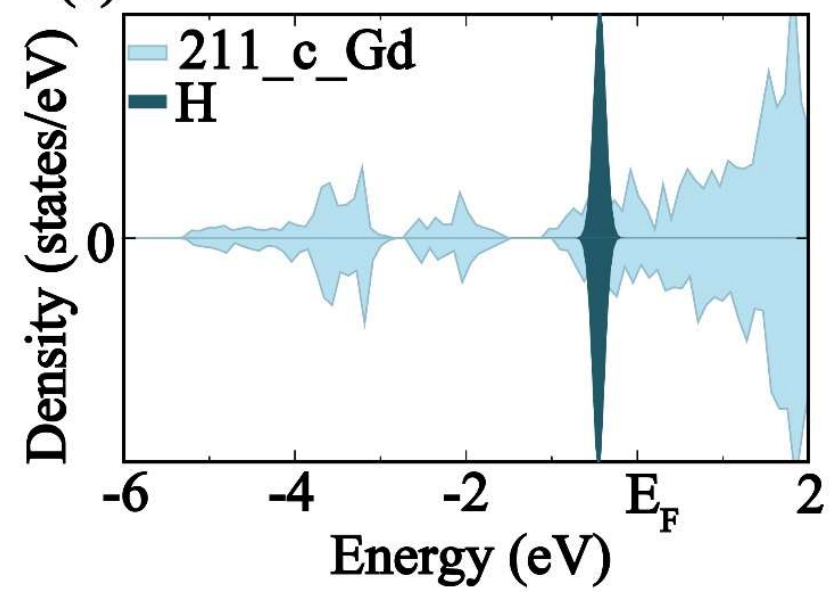

Figure S12: Partial density of states(DOS) for Gd and Te atom on (211) surface 


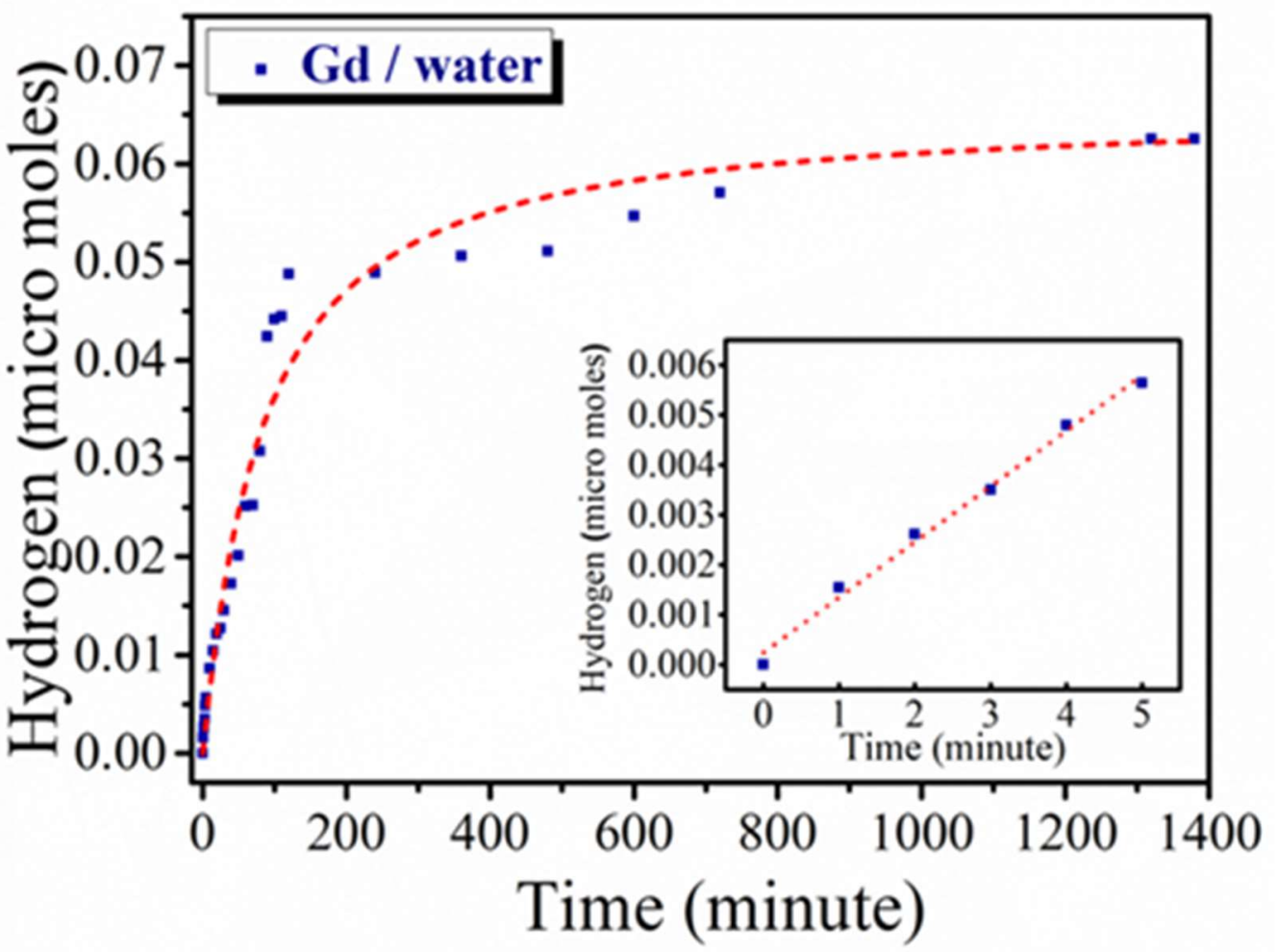

Figure S13: Hydrogen generation as a function of time for Gd in water. The inset image shows the evolution of gas for initial minutes to calculate rate of reaction. A Logistic and linear fit of the data recorded is shown as red dashed line.

Table S1: Adsorption energy, Free energy corrections, Free energy, Average activity, Band centre for different active sites. 
Table S1.

\begin{tabular}{|c|c|c|c|c|c|}
\hline $\begin{array}{l}\text { Adsorption } \\
\text { site }\end{array}$ & $\begin{array}{l}\text { Adsorption } \\
\text { energy }(\mathrm{eV})\end{array}$ & $\begin{array}{l}\Delta(\mathrm{ZPE}-\mathrm{TS}) \\
(\mathrm{eV})\end{array}$ & $\begin{array}{ll}\text { Free } & \text { Energy } \\
(\mathrm{eV}) & \end{array}$ & $\frac{\sum_{\mathrm{n}} \Delta \mathrm{G}_{\mathrm{H}}^{0}(\mathrm{n})}{\sum_{\mathrm{n}} \mathrm{n}}(\mathrm{eV})$ & Band centre $(\mathrm{eV})$ \\
\hline 100_Te & 1.4599 & -0.0256 & 1.4343 & \multirow[t]{2}{*}{0.7488} & -3.05 \\
\hline 100_Gd & 0.1181 & -0.0548 & 0.0633 & & 0.11 \\
\hline 110_Te & 1.7844 & -0.0705 & 1.7139 & \multirow[t]{2}{*}{0.9293} & -2.70 \\
\hline 110_Gd & 0.1915 & -0.0468 & 0.1447 & & 0.19 \\
\hline 111_Te & 0.9034 & -0.0112 & 0.8922 & \multirow[t]{2}{*}{1.0238} & -1.79 \\
\hline 111_Gd & 1.2117 & -0.0563 & 1.1554 & & 0.37 \\
\hline 211_s_Te & 1.325 & -0.0204 & 1.3045 & \multirow{5}{*}{0.93116} & -2.16 \\
\hline 211_s_Gd & -1.0352 & -0.0221 & 1.0573 & & 0.00 \\
\hline 211_t_Te & 1.1516 & -0.0168 & 1.1348 & & -2.00 \\
\hline 211_t_Gd & 0.9649 & -0.0214 & 0.9435 & & -0.05 \\
\hline 211_c_Gd & 0.1709 & 0.0448 & 0.2157 & & -0.07 \\
\hline
\end{tabular}




\section{References:}

1. G. Kresse, J. Hafner, Ab initio molecular dynamics for liquid metals. Phys. Rev. B. 47, 558-561 (1993).

2. J. P. Perdew, K. Burke, M. Ernzerhof, Generalized Gradient Approximation Made Simple. Phys. Rev. Lett. 77, 3865-3868 (1996).

3. A. Jain, S. P. Ong, G. Hautier, W. Chen, W. D. Richards, S. Dacek, S. Cholia, D. Gunter, D. Skinner, G. Ceder, K. a. Persson, The Materials Project: A materials genome approach to accelerating materials innovation. APL Mater. 1, 011002 (2013).

4. R. Kumar, D. Das, A. K. Singh, $\mathrm{C}_{2} \mathrm{~N} / \mathrm{WS}_{2}$ van der Waals type-II heterostructure as a promising water splitting photocatalyst. J. Catal. 359, 143-150 (2018).

5. B. Hinnemann, P. G. Moses, J. Bonde, K. P. Jørgensen, J. H. Nielsen, S. Horch, I. Chorkendorff, J. K. Nørskov, Biomimetic Hydrogen Evolution: $\mathrm{MoS}_{2}$ Nanoparticles as Catalyst for Hydrogen Evolution. J. Am. Chem. Soc. 127, 5308-5309 (2005).

6. A. J. Medford, A. Vojvodic, J. S. Hummelshøj, J. Voss, F. Abild-Pedersen, F. Studt, T. Bligaard, A. Nilsson, J. K. Nørskov, From the Sabatier principle to a predictive theory of transition-metal heterogeneous catalysis. J. Catal. 328, 36-42 (2015).

7. B. Hammer, J. K. Nørskov, in Advances in Catalysis (Academic Press, 2000; https://www.sciencedirect.com/science/article/pii/S0360056402450134), vol. 45 of Impact of Surface Science on Catalysis, pp. 71-129. 